\title{
Monetary Policy and Bubbles in a New Keynesian Model with Overlapping Generations
}

\author{
By JORDI GALî́
}

\begin{abstract}
I analyze an extension of the New Keynesian model that features overlapping generations of finitely lived agents and (stochastic) transitions to inactivity. In contrast with the standard model, the proposed framework allows for the existence of rational expectations equilibria with asset price bubbles. I study the conditions under which bubble-driven fluctuations may emerge and the type of monetary policy rules that may prevent them. I conclude by discussing some of the model's welfare implications. (JEL E12, E32, E44, E52, E63)
\end{abstract}

T

he rise and collapse of speculative bubbles are viewed by many economists and policymakers as an important source of macroeconomic instability and a challenge for monetary policy. ${ }^{1}$ Yet the recurrent reference to bubbles in the policy debate contrasts with their conspicuous absence in modern monetary models. A likely explanation for this seeming anomaly lies in the fact that standard versions of the New Keynesian model, the workhorse framework used in monetary policy analysis, leave no room for the existence of bubbles in equilibrium and hence for any meaningful model-based discussion of their possible interaction with monetary policy. ${ }^{2}$

In the present paper, I develop a modified version of the New Keynesian model, which allows, under certain conditions, for the emergence of bubble-driven

\footnotetext{
* CREI, Universitat Pompeu Fabra and Barcelona GSE, Ramon Trias Fargas 25, 08005 Barcelona, Catalonia, Spain (email: jgali@crei.cat). Giorgio Primiceri was coeditor for this article. I am grateful for comments from Davide Debortoli, Alberto Martín, Jaume Ventura, Michael Reiter, Orazio Attanasio, Gadi Barlevy, Óscar Arce, Franck Portier, Sergi Basco, Anton Nakov, Luca Dedola, Roberto Billi, Camilo Marchesini, three anonymous referees, and conference and/or seminar participants at CREI, NBER Summer Institute, University of Mannheim, Rome MBF Conference, Seoul National University, University of Vienna, New York University, Columbia University, Bank of Spain first Annual Research conference, EEA Lisbon Congress, CEPR ESSIM, Barcelona GSE Summer Forum, first Catalan Economic Society Conference, University College London ADEMU Conference, and 22nd Spring Meeting of Young Economists (Halle). Ángelo Gutiérrez, Christian Hoynck, and Cristina Manea provided excellent research assistance. I acknowledge the European Research Council for financial support under the European Union's Seventh Framework Programme (FP7/2007-2013, ERC Grant agreement no. 339656). I am also thankful for generous financial support from the CERCA Programme/Generalitat de Catalunya and the Severo Ochoa Programme for Centres of Excellence in R\&D.

${ }^{\dagger}$ Go to https://doi.org/10.1257/mac.20180427 to visit the article page for additional materials and author disclosure statement( $\mathrm{s}$ ) or to comment in the online discussion forum.

${ }^{1}$ See, e.g., Borio and Lowe (2002) for an early statement of the risks posed by asset price fluctuations and the need for an appropriate monetary policy response. Taylor (2014) points to excessively low interest rates in the 2000s as a factor behind the housing boom that preceded the financial crisis of 2007-2008. See also Barlevy (2018) for a discussion of the contrast between economists' and policymakers' perspectives on bubbles.

${ }^{2}$ The reason is well known: the equilibrium requirement that the bubble grows at the rate of interest violates the transversality condition of the infinite-lived representative consumer assumed in the New Keynesian model (and in most macro models). See, e.g., Santos and Woodford (1997) and further discussion below.
} 
fluctuations in equilibrium. The modifications to the standard model involve the introduction of overlapping generations (OLG) of finitely lived consumers and stochastic transitions to inactivity (retirement). ${ }^{3}$ For brevity, I henceforth refer to the proposed framework as the OLG-NK model. The assumption of an infinite sequence of cohorts makes it possible for the transversality condition of any individual consumer to be satisfied in equilibrium, even in the presence of a bubble that grows at the rate of interest. ${ }^{4}$ Furthermore, the assumption of retirement (or, more generally, of a relative decline of individual labor income over the life cycle) can generate an equilibrium rate of interest that does not exceed the economy's trend growth rate, a necessary condition for the size of the bubble to remain bounded relative to the size of the economy. Finally, the assumption of sticky prices-a key feature of the New Keynesian model — has two important implications that are missing in most models with bubbles found in the literature. Firstly, price stickiness makes it possible for an aggregate bubble to influence aggregate demand and, hence, output and employment. Secondly, price stickiness makes monetary policy nonneutral, thus allowing for a meaningful discussion of the potential role of monetary policy when the possibility of bubbles and bubble-driven fluctuations is part of the environment. An appealing feature of the OLG-NK framework proposed here is that it nests the standard New Keynesian model (the NK model, henceforth) as a limiting case, when the probabilities of death and retirement approach zero. ${ }^{5}$

After deriving the equations describing the model's equilibrium, I characterize the (perfect foresight) balanced growth paths consistent with that equilibrium and discuss the conditions under which a (nonvanishing) bubble may exist along those paths. If the incidence of retirement is sufficiently low (relative to the consumer's discount rate), there exists a unique balanced growth path, and it is a bubbleless one (as in the standard model). On the other hand, if the probability of retirement is sufficiently high (but plausibly so), a multiplicity of bubbly balanced growth paths is shown to exist (each characterized by a different bubble-output ratio) in addition to a bubbleless one (which always exists).

After characterizing the existence and potential multiplicity of balanced growth paths - bubbly and bubbleless - I turn to the analysis of the stability properties of those paths and the role of monetary policy in shaping those properties. In order to do so, I log-linearize the equilibrium conditions around a balanced growth path as in the standard analysis of the textbook NK model, underscoring throughout the tractability of the new framework.

Several findings of interest emerge from the analysis of the equilibrium dynamics of the OLG-NK model.

Firstly, the introduction of an overlapping generations structure by itself does not change any of the qualitative properties of the standard NK model. Thus, in the

\footnotetext{
${ }^{3}$ Other authors recently have extended the New Keynesian model to incorporate overlapping generations of finite-lived agents into the New Keynesian framework, though none of them has investigated the possible existence of bubbles. See the literature discussion below.

${ }^{4}$ And even though that transversality condition does not hold for the economy as a whole.

${ }^{5}$ In Galí (2014) I also analyzed an OLG model with sticky prices and bubbles. That model, however, did not allow for the possibility of bubble-driven fluctuations in output, nor did it nest the standard New Keynesian model. See the literature review below.
} 
absence of bubbles, the resulting equilibrium conditions describing the dynamics of inflation and the output gap are isomorphic to those of the standard model with a "modified" discount factor. Among other properties, the so-called "forward-guidance puzzle" carries over unaltered to the OLG-NK framework despite the introduction of finitely lived consumers.

Secondly, and most importantly, the OLG-NK structure allows for the existence, under certain conditions, of bubble-driven fluctuations. A "leaning against the bubble" (LAB, henceforth) interest rate policy, if precisely calibrated, may succeed in insulating output and inflation from aggregate bubble fluctuations. Furthermore, if aggressive enough, the LAB policy may be able to rule out bubble fluctuations themselves, with the required strength of the response depending in a nonlinear way on the value of the bubble-output ratio along the associated balanced growth path. On the other hand, an LAB policy which does not succeed in eliminating bubble fluctuations may end up increasing the volatility and persistence of bubble fluctuations as in Galí (2014).

Thirdly, a policy that targets the output gap (or inflation) while ignoring the bubble will generally succeed at stabilizing those macro variables. On the other hand, only if the average size of the bubble-output ratio lies above a certain threshold, a sufficiently aggressive output-based stabilization policy will succeed in ruling out fluctuations in the bubble as well.

Finally, and with regard to the welfare implications of the OLG-NK model, two main results are derived. First, expected lifetime utility along a deterministic balanced growth path (BGP) is increasing in the associated bubble-output ratio. Secondly, an increase (decrease) in the size of the aggregate bubble raises (lowers) the expected lifetime utility of all cohorts as long as monetary policy doesn't "overreact" to it. However, and to the extent that consumers are risk averse, recurrent symmetric bubble-driven fluctuations will be welfare reducing and will thus justify policies that eliminate them. In sum: large bubbles are good, but their fluctuations are generally not (unless they imply larger bubbles as in one of the examples analyzed).

The paper concludes with some reflections on the caveats and limitations of the OLG-NK model developed here and points to some directions for future research. An important caveat of the present model, worth emphasizing at the outset, is that bubble fluctuations have an impact on the economy mainly as aggregate demand shifters. ${ }^{6}$ In particular, they do not generate any distortion that would break down the "divine coincidence" property, which still holds in the OLG-NK economy. Accordingly, the role of monetary policy is either to offset the effects of bubbles on aggregate demand or to prevent the emergence of bubble fluctuations altogether. In both cases, the central bank aims at stabilizing the output gap and inflation. As I discuss in the concluding section, one can think of extensions of the basic model developed here where the emergence of a bubble may generate a trade-off calling for a more nuanced policy response.

The rest of the paper is organized as follows. Section I summarizes the related literature. Section II describes the basic framework underlying the analysis in the rest of the paper. Section III characterizes the economy's balanced growth paths.

\footnotetext{
${ }^{6}$ I write "mainly" because, as discussed below, they may also have an impact on the distribution of wealth.
} 
Section IV analyzes the potential role of bubbles as a source of fluctuations and discusses the consequences of alternative policies in that regard. Section V makes some considerations about the welfare implications of bubbles and bubble-driven fluctuations. Section VI summarizes and concludes.

\section{Related Literature}

Much of the literature on rational bubbles in general equilibrium has been based on real models. ${ }^{7}$ An early reference in that category is Tirole (1985), using a conventional overlapping generations framework with capital accumulation. More recently, Farhi and Tirole (2011), Martin and Ventura (2012), Miao and Wang (2012, 2014, 2018), Basco (2014), Aoki and Nikolov (2015), Hirano and Yanagawa (2017), Bengui and Phan (2018), and Ikeda and Phan (2019), among others, have studied the role of bubbles in real models with financial frictions, in which bubbles interact with financial constraints, generally relaxing them.

There is also an extensive literature on bubbles using monetary models with fully flexible prices. In most of those models, including the seminal paper by Samuelson (1958), money itself is the bubbly asset. Asriyan et al. (2016) provide a more recent example, introducing the notion of a "nominal" bubble. While monetary policy is not always neutral in those models, the mechanism through which its effects are transmitted is very different from that emphasized in models with nominal rigidities.

A number of papers have modified the standard NK model by introducing overlapping generations à la Blanchard-Yaari, though none of them has considered the possibility of bubbles. Piergallini (2006) develops a related model with money in the utility function to analyze the implications of the real balance effect on the stability properties of interest rate rules. Nisticò (2012) discusses the desirability of a systematic monetary policy response to stock price developments in a similar model but in the absence of bubbles. Del Negro, Giannoni, and Patterson (2015) propose a related framework as a possible solution to the "forward guidance puzzle." None of the previous authors allow for retirement or declining labor income in their frameworks. That feature plays a central role in the emergence of asset price bubbles in the model proposed here.

Bernanke and Gertler $(1999,2001)$ analyze the possible gains from "leaning against the wind" monetary policies in an NK model in which stock prices contain an ad hoc deviation from their fundamental value. The properties of that deviation differ from those of a rational bubble, which cannot exist in their model, which assumes an infinitely lived representative consumer.

In Galí (2014), I studied the interaction between rational bubbles and monetary policy in a two-period overlapping generations model with sticky prices, emphasizing some of the risks associated with "leaning against the bubble" policies. 8 While closest in spirit to the present paper, the framework used in that paper had

\footnotetext{
${ }^{7}$ See Martin and Ventura (2018) and Miao (2014) for recent surveys on the theory of bubbles.

${ }^{8}$ See also the comment by Miao, Shen, and Wang (2019) for an analysis of e-learnability of the bubbly equilibria in Galí (2014), around both a stable and an unstable steady state.
} 
important limitations. In particular, it ruled out the possibility of bubble-driven fluctuations since employment and output were constant in equilibrium, with the bubble only having redistributive effects. By contrast, the model developed here shows how bubble fluctuations, in combination with price stickiness, can have an impact on economic activity, with an explicit analysis of the mechanisms that determine the size of that impact (i.e., the "bubble multiplier"). On the other hand, the assumption of two-period lived individuals made in Galí (2014), while convenient, cannot be easily reconciled with the frequency of observed asset boom-bust episodes (not to say with the observed duration of individual prices). By way of contrast, the model developed here is consistent with a calibration to a quarterly frequency (as is conventional in the business cycle literature) and could thus be potentially embedded in a quantitative medium-scale DSGE model. Finally, it is worth noting that the structure of the linearized equilibrium conditions of the OLG-NK model developed below is isomorphic to that of the familiar textbook NK model, with the aggregate bubble being now a driver of aggregate demand whose evolution is described by an additional equation. Some special cases of interest nested in the OLG-NK model below include (i) the standard NK model itself, (ii) an economy with finite lives and retirement but no bubbles, and (iii) an economy with a constant bubble-output ratio. The use of those special cases as benchmarks facilitates the understanding of the factors driving the properties of the OLG-NK economy. Several insights emerge from that analysis, discussed in detail below, pertaining to the persistence of the "forward guidance puzzle" in the presence of finite horizons and the role played by different factors in strengthening or weakening the requirements on the policy rule in order to guarantee a unique equilibrium.

Two recent working papers have explored, using alternative perspectives, the connection between monetary policy and asset bubbles. In contrast with the present contribution, however, both papers involve frameworks characterized by a monetary transmission mechanism very different from that found in the standard NK model. Thus, Allen, Barlevy, and Gale (2017) revisit the relationship between interest rates and asset bubbles discussed in Galí (2014) using a variety of frameworks (mostly nonmonetary) and exploring the conditions and environments that determine the sign of that relation. Dong, Miao, and Wang (2018) analyze the implications of alternative monetary policy rules (including rules that respond systematically to asset bubbles) in an NK model with infinitely lived agents and where a bubbly asset can help alleviate entrepreneurs' funding constraints. Monetary policy affects the amount of liquidity in the economy — and, as a result, the value attached to the bubbly asset - through the impact of inflation on real reserves. They show how in that framework, a "leaning against the bubble" policy may reduce bubble volatility but possibly at the cost of raising inflation volatility.

Finally, my paper is also related to Caballero and Simsek (2020), which studies a New Keynesian economy in which asset valuations, partly driven by investors' heterogeneous beliefs, are an important determinant of aggregate demand and output. The authors examine the role the both monetary and macroprudential policies can play at dampening the resulting excessive fluctuations in asset valuations and output. 


\section{A New Keynesian Model with Overlapping Generations}

Next, I describe the basic framework underlying the analysis in the rest of the paper.

\section{A. Consumers}

I assume an economy with overlapping generations of the "perpetual youth" type as in Yaari (1965) and Blanchard (1985). The size of the population is constant and normalized to one. Each individual has a constant probability $\gamma$ of surviving into the following period, independently of his age and economic status ("active" or "retired"). A cohort of size $1-\gamma$ is born (in an economic sense) and becomes active each period. Thus, the size in period $t \geq s$ of the cohort born in period $s$ is given by $(1-\gamma) \gamma^{t-s}$.

At any point in time, two types of individuals coexist in the economy, "active" and "inactive." Active individuals supply labor and manage their own firms, which they set up when they are born. I assume that each active individual faces a constant probability $1-v$ of becoming "inactive," i.e., of permanently losing his job and quitting his entrepreneurial activities. For concreteness, below I refer to that transition as "retirement," though it should be clear that it can be given a broader interpretation. ${ }^{9}$ The previous assumptions imply that the size of the active population (and, hence, the measure of firms) at any point in time is constant and given by $\alpha \equiv(1-\gamma) /(1-v \gamma) \in(0,1]$.

A representative consumer from cohort $s$ chooses a consumption plan to maximize expected lifetime utility

$$
E_{s} \sum_{t=s}^{\infty}(\beta \gamma)^{t-s} \log C_{t \mid s}
$$

subject to the sequence of period budget constraints

$$
\frac{1}{P_{t}} \int_{0}^{\alpha} P_{t}(i) C_{t \mid s}(i) d i+E_{t}\left\{\Lambda_{t, t+1} Z_{t+1 \mid s}\right\}=A_{t \mid s}+W_{t} N_{t \mid s}
$$

for $t=s, s+1, s+2, \ldots$ The term $\beta \equiv 1 /(1+\rho) \in(0,1)$ is the discount factor, $C_{t \mid s} \equiv\left(\alpha^{-\frac{1}{\epsilon}} \int_{0}^{\alpha} C_{t \mid s}(i)^{1-\frac{1}{\epsilon}} d i\right)^{\frac{\epsilon}{\epsilon-1}}$ is a consumption index, $C_{t \mid s}(i)$ is the quantity purchased of good $i \in[0, \alpha]$ at a price $P_{t}(i)$, and $P_{t} \equiv\left(\alpha^{-1} \int_{0}^{\alpha} P_{t}(i)^{1-\epsilon} d i\right)^{\frac{1}{1-\epsilon}}$ is the price index.

Complete markets for state-contingent securities are assumed, with $Z_{t+1 \mid s}$ denoting the stochastic payoff at $t+1$ (expressed in units of the consumption index) generated by the portfolio of securities purchased in period $t$. The market value of that portfolio is given by $E_{t}\left\{\Lambda_{t, t+1} Z_{t+1 \mid s}\right\}$, where $\Lambda_{t, t+1}$ is the stochastic discount factor for one-period-ahead (real) payoffs. Only individuals who are alive can trade

\footnotetext{
${ }^{9}$ Gertler (1999) introduces retirement in a similar fashion in a model of social security. More recently, Carvalho, Ferrero, and Nechio (2016) have used a version of the Gertler model to analyze the sources of low-frequency changes in the equilibrium real rate. Blanchard (1985) assumes that relative productivity declines with age and analyzes its impact on the real rate. All these papers develop real models, in contrast to the present one, and do not consider the possibility of bubbles.
} 
in securities markets. Note that the existence of complete securities markets allows individuals to insure against the "risk" of retirement.

Variable $A_{t \mid s}$ denotes financial wealth at the start of period $t$ for a member of cohort $s \leq t$. For individuals other than those born in the current period, $A_{t \mid s}=Z_{t \mid s} / \gamma$, where the term $1 / \gamma$ captures the additional return on wealth resulting from an annuity contract. As in Blanchard (1985), that contract has the holder receive each period from a (perfectly competitive) insurance firm an annuity payment proportional to his financial wealth, in exchange for transferring that wealth to the insurance firm upon death. 10

Variable $W_{t}$ denotes the (real) wage per hour, and $N_{t \mid s}$ individual work hours for a member of cohort $s \leq t$. Both the wage and work hours are taken as given by each worker. Each firm determines the work hours it wants to hire given desired output and technology. Aggregate work hours, $N_{t}$, are allocated uniformly among all active individuals, i.e., $N_{t \mid s}^{a}=N_{t} / \alpha .{ }^{11}$ Note that $N_{t \mid s}^{r}=0$ for retired individuals. Normalizing an individual's time endowment to unity, it must be the case that $N_{t} \leq \alpha$ for all $t$, which I assume throughout.

Finally, I assume a solvency constraint of the form $\lim _{T \rightarrow \infty} \gamma^{T} E_{t}\left\{\Lambda_{t, t+T} A_{t+T \mid s}\right\} \vec{\nabla}$ 0 for all $t$, where $\Lambda_{t, t+T}$ is determined recursively by $\Lambda_{t, t+T}=\Lambda_{t, t+T-1} \Lambda_{t+T-1, t+T} \cdot{ }^{12}$

The problem above yields a set of optimal demand functions

$$
C_{t \mid s}(i)=\frac{1}{\alpha}\left(\frac{P_{t}(i)}{P_{t}}\right)^{-\epsilon} C_{t \mid s}
$$

for all $i \in[0, \alpha]$, which in turn imply $\int_{0}^{\alpha} P_{t \mid s}(i) C_{t \mid s}(i) d i=P_{t} C_{t \mid s}$. Thus, we can rewrite the period budget constraint as

$$
C_{t \mid s}+\gamma E_{t}\left\{\Lambda_{t, t+1} A_{t+1 \mid s}\right\}=A_{t \mid s}+W_{t} N_{t \mid s}
$$

The consumer's optimal plan must satisfy the optimality condition ${ }^{13}$

$$
\Lambda_{t, t+1}=\beta \frac{C_{t \mid s}}{C_{t+1 \mid s}}
$$

\footnotetext{
${ }^{10}$ Thus, individuals who hold negative assets will pay an annuity fee to the insurance company. The latter absorbs the debt in case of death. The insurance arrangement can also be replicated through securities markets. In that case, the individual will purchase a portfolio that generates a random payoff $A_{t+1 \mid s}$ if he remains alive, zero otherwise. The value of that payoff will be given by $E_{t}\left\{\Lambda_{t, t+1} \gamma A_{t+1 \mid s}\right\}$, which is equivalent to the formulation in the main text, given that $A_{t \mid s}=Z_{t \mid s} / \gamma$.

${ }^{11}$ Note that by assuming an inelastic labor supply and demand-determined work hours, I abstract from a labor supply decision. An alternative setup with labor disutility and a competitive labor market would generate systematic counterfactual differences in the quantity of labor supplied by active individuals across age groups, due to wealth effects. Alternatively, one may assume preferences that rule out those wealth effects but at the cost of rendering the analysis below less tractable.

${ }^{12}$ Note that $(\Lambda \gamma)^{-1}$ is the "effective" (i.e., including the impact of the annuity) interest rate paid by a borrower in the steady state. The solvency constraint thus has the usual interpretation of a no-Ponzi game condition.

${ }^{13}$ Note that in the optimality condition, the survival probability $\gamma$ and the extra return $1 / \gamma$ resulting from the annuity contract cancel each other. Complete markets guarantee the same consumption growth rate between two different periods for all consumers alive in the two periods, including those who are retiring. Thus, individual consumption is equated within each cohort, with differences across cohorts resulting from differences in their relative wealth (financial and human) at birth.
} 
and the transversality condition

$$
\lim _{T \rightarrow \infty} \gamma^{T} E_{t}\left\{\Lambda_{t, t+T} A_{t+T \mid s}\right\}=0
$$

with (4) holding for all possible states of nature (conditional on the individual remaining alive in $t+1)$.

As shown in the Appendix, by combining (3), (4), and (5), one can derive the following consumption functions for active and retired individuals, respectively:

$$
\begin{aligned}
C_{t \mid s}^{a} & =(1-\beta \gamma)\left[A_{t \mid s}^{a}+\frac{1}{\alpha} \sum_{k=0}^{\infty}(v \gamma)^{k} E_{t}\left\{\Lambda_{t, t+k} W_{t+k} N_{t+k}\right\}\right], \\
C_{t \mid s}^{r} & =(1-\beta \gamma) A_{t \mid s}^{r},
\end{aligned}
$$

where the superscript $\{a, r\}$ denotes the consumer's status, active or retired. 14

\section{B. Firms}

Each individual is endowed with the know-how to produce a differentiated good and sets up a firm with that purpose at birth. That firm remains operative until its founder retires or dies, whatever comes first. ${ }^{15}$ All firms have an identical technology, represented by the linear production function

$$
Y_{t}(i)=\Gamma^{t} N_{t}(i)
$$

where $Y_{t}(i)$ and $N_{t}(i)$ denote output and employment for firm $i \in[0, \alpha]$, respectively, and $\Gamma \equiv 1+g \geq 1$ denotes the (gross) rate of productivity growth. Individuals cannot work at their own firms and must hire instead labor services provided by others. 16

Aggregation of (2) across consumers yields the demand schedule facing any given firm:

$$
C_{t}(i)=\frac{1}{\alpha}\left(\frac{P_{t}(i)}{P_{t}}\right)^{-\epsilon} C_{t}
$$

where $C_{t} \equiv(1-\gamma) \sum_{s=-\infty}^{t} \gamma^{t-s} C_{t \mid s}$ is aggregate consumption. Each firm takes as given the aggregate price level $P_{t}$ and aggregate consumption $C_{t}$.

As in Calvo (1983), each firm is assumed to freely set the price of its good with probability $1-\theta$ in any given period, independently of the time elapsed since

\footnotetext{
${ }^{14}$ The assumption of complete markets guarantees that for any given cohort $s, C_{t \mid s}^{a}=C_{t \mid s}^{r}$ for all $t \geq s$. It follows that $A_{t \mid s}^{r}=A_{t \mid s}^{a}+(1 / \alpha) \sum_{k=0}^{\infty}(\gamma v)^{k} E_{t}\left\{\Lambda_{t, t+k} W_{t+k} N_{t+k}\right\}$.

${ }^{15}$ The assumption of finitely lived firms (or more generally, firms whose dividends shrink relative to the size of the economy) is needed in order for bubbles to exist in equilibrium, which requires that the interest rate is no greater than the economy's trend growth rate as shown below. By equating the probability of a firm's survival to that of its owner remaining active, I effectively equate the rate at which dividends and labor income are discounted, which simplifies considerably the analysis below. All the qualitative results discussed below carry over to the case of different rates of "retirement" for firms and individuals but at the cost of more cumbersome algebra.

${ }^{16} \mathrm{I}$ assume that each firm newly set up in any given period inherits the index of an exiting firm.
} 
the last price adjustment. With probability $\theta$, an incumbent firm keeps its price unchanged, while a newly created firm sets a price equal to the economy's average price in the previous period. ${ }^{17}$ Accordingly, the aggregate price dynamics are described by the equation

$$
P_{t}^{1-\epsilon}=\theta P_{t-1}^{1-\epsilon}+(1-\theta)\left(P_{t}^{*}\right)^{1-\epsilon}
$$

where $P_{t}^{*}$ is the price set in period $t$ by firms optimizing their price. ${ }^{18} \mathrm{~A}$ log-linear approximation of the previous difference equation around the zero inflation equilibrium yields (letting lowercase letters denote the logs of the original variables)

$$
p_{t}=\theta p_{t-1}+(1-\theta) p_{t}^{*}
$$

i.e., the current price level is a weighted average of last period's price level and the newly set price, all in logs, with the weights given by the fraction of firms that do not and do adjust prices, respectively.

In both environments, a firm adjusting its price in period $t$ will choose the price $P_{t}^{*}$ that maximizes

$$
\max _{P_{t}^{*}} \sum_{k=0}^{\infty}(v \gamma \theta)^{k} E_{t}\left\{\Lambda_{t, t+k} Y_{t+k \mid t}\left(\frac{P_{t}^{*}}{P_{t+k}}-\mathcal{W}_{t+k}\right)\right\}
$$

subject to the sequence of demand constraints

$$
Y_{t+k \mid t}=\frac{1}{\alpha}\left(\frac{P_{t}^{*}}{P_{t+k}}\right)^{-\epsilon} C_{t+k}
$$

for $k=0,1,2, \ldots$, where $Y_{t+k \mid t}$ denotes output in period $t+k$ for a firm that last reset its price in period $t ; \mathcal{W}_{t} \equiv W_{t} / \Gamma^{t}$ is the productivity-adjusted real wage. ${ }^{19}$ Note that the $(v \gamma)^{k}$ term used in the discounting of future profits corresponds to the probability that the firm remains operative $k$ periods ahead, while $\theta^{k}$ is the probability that the newly set price remains effective $k$ periods ahead. Other than for the additional discounting, the present optimal price-setting problem is identical to that in the standard model, so the reader is referred to Galí (2015) for a discussion and derivation details.

The optimality condition associated with the problem above takes the form

$$
\sum_{k=0}^{\infty}(v \gamma \theta)^{k} E_{t}\left\{\Lambda_{t, t+k} Y_{t+k \mid t}\left(\frac{P_{t}^{*}}{P_{t+k}}-\mathcal{M} \mathcal{W}_{t+k}\right)\right\}=0
$$

where $\mathcal{M} \equiv \epsilon /(\epsilon-1)$ is the optimal markup under flexible prices.

\footnotetext{
${ }^{17}$ Alternatively, a fraction $\theta$ of newly created firms "inherit" the price in the previous period for the good they replace. In either case, I assume a transfer system that equalizes the wealth across members of the new cohort.

${ }^{18}$ Note that the price is common to all those firms since they face an identical problem.

${ }^{19}$ The firm's demand schedule (11) can be derived by aggregating (9) across cohorts.
} 
A first-order Taylor expansion of (12) around the zero inflation balanced growth path yields, after some manipulation,

$$
p_{t}^{*}=\mu+(1-\Lambda \Gamma v \gamma \theta) \sum_{k=0}^{\infty}(\Lambda \Gamma v \gamma \theta)^{k} E_{t}\left\{\psi_{t+k}\right\},
$$

where $\psi_{t} \equiv \log P_{t} \mathcal{W}_{t}$ is the $(\log )$ nominal marginal cost, $\mu \equiv \log \mathcal{M}$, and $\Lambda \equiv 1 /(1+r)$ is the steady-state stochastic discount factor, with $r$ being the steady-state real interest rate. Throughout, I maintain the assumption that $\Lambda \Gamma v \gamma \in[0,1)$, which guarantees that the firm's problem is well defined in a neighborhood of the zero inflation balanced growth path.

Letting $\mu_{t} \equiv p_{t}-\psi_{t}=-\log \mathcal{W}_{t}$ denote the average (log) price markup and combining (10) and (13) yields the inflation equation:

$$
\pi_{t}=\Lambda \Gamma v \gamma E_{t}\left\{\pi_{t+1}\right\}-\lambda\left(\mu_{t}-\mu\right)
$$

where $\pi_{t} \equiv p_{t}-p_{t-1}$ denotes inflation and $\lambda \equiv(1-\theta)(1-\Lambda \Gamma v \gamma \theta) / \theta>0 .{ }^{20}$

The details of wage setting are not central to the main point of the paper. As noted above, work hours are demand determined and allocated uniformly among active individuals. ${ }^{21}$ For convenience, I assume an ad hoc wage schedule linking the productivity-adjusted real wage $\mathcal{W}_{t}$ to work hours per capita:

$$
\mathcal{W}_{t}=\left(\frac{N_{t}}{\alpha}\right)^{\varphi}
$$

where $N_{t} \equiv \int_{0}^{\alpha} N_{t}(i) d i$ denotes aggregate work hours and $\alpha$ is the aggregate labor supply. The wage is taken as given by firms. The previous wage schedule implies a countercyclical price markup, $\mu_{t}-\mu=-\varphi \log \left(N_{t} / N\right)$.

Wage schedule (15), together with the assumption of a constant gross markup $\mathcal{M}$ under flexible prices and production function (8), implies a natural (i.e., flexible price) level of output given by $Y_{t}^{n}=\Gamma^{t} \mathcal{Y}$, where $\mathcal{Y} \equiv \alpha \mathcal{M}^{-\frac{1}{\varphi}}$. The previous expression also corresponds to aggregate hours under flexible prices. Note that as long as firms exercise their market $\operatorname{power}(\mathcal{M}>1)$, aggregate hours under flexible prices will be less than the available aggregate time endowment $\alpha$. This will also be true in the sticky price equilibrium, as long as fluctuations are "small." The fact that the natural level of output follows a deterministic trend is, of course, a consequence of the (deliberate) absence of fundamental shocks in the framework above. But it implies a convenient property of the present model: bubble-driven fluctuations in output and employment cannot emerge under fully flexible prices since in that case those variables are pinned down by the supply block of the model.

\footnotetext{
${ }^{20}$ Note that in the standard NK model with a representative consumer, the coefficient on expected inflation is given by $\beta$, while the slope coefficient is $\lambda \equiv(1-\theta)(1-\beta \theta) / \theta$. Those expressions correspond to the limit of the expressions in the text as $v \gamma \rightarrow 1$ and given that $\Lambda \Gamma=\beta$ along a balanced growth path under the assumption of an infinitely lived representative consumer with log utility.

${ }^{21}$ The alternative of a perfectly competitive labor market, combined with an inelastic labor supply at the individual level, would imply constant aggregate employment and rule out the possibility of bubble-driven fluctuations. On the other hand, introducing labor disutility would lead to a dispersion in hours worked across cohorts driven by a wealth effect, an implication that is likely counterfactual and would complicate the analysis substantially.
} 
Log-linearizing (15) and combining the resulting expressions with (14), we obtain a version of the New Keynesian Phillips curve:

$$
\pi_{t}=\Lambda \Gamma v \gamma E_{t}\left\{\pi_{t+1}\right\}+\kappa \hat{y}_{t},
$$

where $\kappa \equiv \lambda \varphi$ and $\hat{y}_{t} \equiv \log \left(Y_{t} / Y_{t}^{n}\right)$ is the output gap. Two remarks are worth making with regard to the properties of (16). Firstly, one should note that in contrast with the standard NK model, the coefficient on expected inflation is not pinned down by the consumer's discount factor. Instead, it depends on parameters affecting the life expectancy of firms (through $v \gamma$ ) as well as the gap between the real interest rate and the growth rate along a balanced growth path (as captured by $\Lambda \Gamma$ ), all of which determine the effective "forward-lookingness" of price-setting. In contrast with the standard model, however, and as discussed below, the interest rate along a balanced growth path, $r=\Lambda^{-1}-1$, is not always uniquely determined by primitive parameters and may be instead related to the size of the bubble along a balanced growth path. Secondly, the assumed environment and, in particular, the absence of time-varying distortions on the supply side (related or not to the presence of a bubble) imply the absence of a trade-off between stabilization of inflation and the output gap; i.e., the "divine coincidence" holds in the present model as is clear from (16). In other words, the macroeconomic effects of bubble fluctuations in the model are restricted to work through its impact on aggregate demand. Things would likely be different in an environment with financial frictions in which bubbles affected borrowing constraints for firms' working capital and/or distorted a consumption-investment decision, thus implying an inefficient allocation even under flexible prices. The analysis of such an extension is beyond the scope of the present paper. 22

\section{Asset Markets}

In addition to annuity contracts and a complete set of state-contingent securities, I assume the existence of markets for some other specific assets, whose prices and returns must satisfy certain equilibrium conditions. In particular, the yield on a one-period nominally riskless bond purchased in period $t$, denoted by $i_{t}$, must satisfy ${ }^{23}$

$$
1=\left(1+i_{t}\right) E_{t}\left\{\Lambda_{t, t+1} \frac{P_{t}}{P_{t+1}}\right\},
$$

thus implying that the relation $\Lambda \equiv 1 /(1+r)$ between the discount factor and the real return on a riskless nominal bond $(r)$ will hold along a perfect foresight balanced growth path.

\footnotetext{
${ }^{22}$ Note, however, that the Phillips curve is not completely independent from the presence of a bubble since the latter's size along a balanced growth path will generally affect the real interest rate and, hence, discount factor $\Lambda$.

${ }^{23}$ Note also that in the asset pricing equations, and from the viewpoint of an individual investor, the probability of remaining alive $\gamma$ and the extra return $1 / \gamma$ resulting from the annuity contract cancel each other.
} 
Stocks in individual firms trade at a price (before dividends) $Q_{t}^{F}(i)$, for all $i \in[0, \alpha]$, which must satisfy the asset pricing equation

$$
Q_{t}^{F}(i)=D_{t}(i)+v \gamma E_{t}\left\{\Lambda_{t, t+1} Q_{t+1}^{F}(i)\right\},
$$

where $D_{t}(i) \equiv Y_{t}(i)\left(\left(P_{t}(i) / P_{t}\right)-\mathcal{W}_{t}\right)$ denotes firm $i$ 's dividends and $v \gamma$ is the probability that firm $i$ survives into next period. Solving (18) forward under the assumption that $\lim _{k \rightarrow \infty}(v \gamma)^{k} E_{t}\left\{\Lambda_{t, t+k} Q_{t+k}^{F}(i)\right\}=0$ and aggregating across firms,

$$
Q_{t}^{F} \equiv \int_{0}^{\alpha} Q_{t}^{F}(i) d i=\sum_{k=0}^{\infty}(v \gamma)^{k} E_{t}\left\{\Lambda_{t, t+k} D_{t+k}\right\}
$$

where $D_{t} \equiv \int_{0}^{\alpha} D_{t}(i) d i$ denotes aggregate dividends. Note that the fact that individual firms are finitely lived makes it possible for the aggregate value of currently traded firms to be finite even if the interest rate is lower than the growth rate of aggregate dividends.

Much of the analysis below focuses on intrinsically worthless assets-i.e., assets generating no dividend, pecuniary or not-which may yet be traded at a positive price, constituting a pure bubble. ${ }^{24}$ Let $Q_{t}^{B}$ denote the aggregate value of bubble assets in period $t$. In equilibrium, that variable evolves over time according to the following two equations:

$$
\begin{aligned}
& Q_{t}^{B}=U_{t}+B_{t}, \\
& Q_{t}^{B}=E_{t}\left\{\Lambda_{t, t+1} B_{t+1}\right\},
\end{aligned}
$$

where $U_{t} \equiv Q_{t \mid t}^{B} \geq 0$ is the value in period $t$ of new bubbles introduced by the newly born cohort ${ }^{25}$ and $B_{t} \equiv \sum_{s=-\infty}^{t-1} Q_{t \mid s}^{B} \geq 0$ is the aggregate value in period $t$ of preexisting bubble assets, already available for trade in period $t-1$, with $Q_{t \mid s}^{B}$ denoting the period $t$ value of bubble assets introduced in period $s \leq t$. The nonnegativity constraints follow from the assumption of free disposal. Note also that the introduction of new bubble assets by incoming cohorts makes it possible for an aggregate bubble to reemerge after a hypothetical collapse, thus overcoming a common criticism of early models of rational bubbles. A similar environment with bubble creation was first introduced and analyzed in Martin and Ventura (2012) in the context of an overlapping generations model with capital accumulation and financial frictions. ${ }^{26}$

\footnotetext{
${ }^{24}$ In Jean Tirole's words, pure bubbly assets are "best thought of as pieces of paper."

${ }^{25}$ Think of pieces of paper of a cohort-specific color or stamped with the birth year of their originators.

${ }^{26}$ One possible interpretation is that the bubble introduced by each individual is "attached" to the stock of his firm, and, hence, it bursts whenever the firm stops operating (i.e., with probability $1-v \gamma$ ). See below for an example of such a stochastic bubble.
} 
Note that in the previous environment, the initial financial wealth of a member of a cohort born in period $t$ is given by

$$
A_{t \mid t}=Q_{t \mid t}^{F}+U_{t} /(1-\gamma)
$$

where $Q_{t \mid t}^{F}$ is the value in period $t$ of a newly created firm. ${ }^{27}$

\section{Market Clearing}

Goods market clearing requires $Y_{t}(i)=(1-\gamma) \sum_{s=-\infty}^{t} \gamma^{t-s} C_{t \mid s}(i)$ for all $i \in[0, \alpha]$. Letting $Y_{t} \equiv\left(\alpha^{-\frac{1}{\epsilon}} \int_{0}^{\alpha} Y_{t}(i)^{1-\frac{1}{\epsilon}} d i\right)^{\frac{\epsilon}{\epsilon-1}}$ denote aggregate output, we have

$$
Y_{t}=(1-\gamma) \sum_{s=-\infty}^{t} \gamma^{t-s} C_{t \mid s}=C_{t}
$$

Note also that in equilibrium

$$
N_{t}=\int_{0}^{\alpha} N_{t}(i) d i=\Delta_{t}^{p} \mathcal{Y}_{t} \simeq \mathcal{Y}_{t}
$$

where $\mathcal{Y}_{t} \equiv Y_{t} / \Gamma^{t}$ is aggregate output normalized by productivity, and $\Delta_{t}^{p} \equiv(1 / \alpha) \int_{0}^{\alpha}\left(P_{t}(i) / P_{t}\right)^{-\epsilon} d i \simeq 1$ is an index of relative price distortions that, up to a first-order approximation, equals one near a zero inflation balanced growth path.

With all securities other than stocks and bubbly assets being in zero net supply, asset market clearing requires

$$
(1-\gamma) \sum_{s=-\infty}^{t} \gamma^{t-s}\left(v^{t-s} A_{t \mid s}^{a}+\left(1-v^{t-s}\right) A_{t \mid s}^{r}\right)=Q_{t}^{F}+Q_{t}^{B} .
$$

Aggregation of consumption functions (6) and (7) across individuals and cohorts, combined with asset market clearing condition (22) and the expression for fundamental financial wealth (19), yields the aggregate consumption function

$$
C_{t}=(1-\beta \gamma)\left(Q_{t}^{B}+X_{t}\right)
$$

where

$$
X_{t} \equiv \sum_{k=0}^{\infty}(v \gamma)^{k} E_{t}\left\{\Lambda_{t, t+k} Y_{t+k}\right\}
$$

can be interpreted as total fundamental wealth (financial plus human), i.e., the discounted sum of current and future income expected to accrue to currently alive consumers.

Next, I characterize the economy's perfect foresight balanced growth paths consistent with zero inflation.

\footnotetext{
${ }^{27} Q_{t \mid t}^{F}$ should be interpreted as net of transfers assumed in order to equate the value of entering firms independently of whether they optimize or not their initial price.
} 


\section{Balanced Growth Paths}

In a perfect foresight balanced growth path (henceforth, BGP), the discount factor is constant and satisfies $\Lambda=1 /(1+r)$, as implied by (17), where $r$ is the corresponding real interest rate. Note also that zero inflation requires that actual and desired markups coincide, i.e., $\mathcal{W}=1 / \mathcal{M}$. Combined with (15), the previous condition implies that output along the BGP is given by $Y_{t}^{B G P}=\Gamma^{t} \mathcal{Y}$, which coincides with the natural level of output as derived above.

Evaluating (23) and (24) at a BGP yields

$$
\mathcal{C}=(1-\beta \gamma)\left[\mathcal{Q}^{B}+\frac{1}{1-\Lambda \Gamma v \gamma} \mathcal{Y}\right],
$$

where $\mathcal{C}$ and $\mathcal{Q}^{B}$ denote aggregate consumption and the aggregate bubble, both evaluated at the BGP and normalized by productivity $\Gamma^{t}$. Goods market clearing requires that $\mathcal{C}=\mathcal{Y}$, thus implying the following equation relating the bubble-output ratio $q^{B} \equiv \mathcal{Q}^{B} / \mathcal{Y}$ and the discount factor $\Lambda$ along a BGP:

$$
1=(1-\beta \gamma)\left[q^{B}+\frac{1}{1-\Lambda \Gamma v \gamma}\right],
$$

or, equivalently,

$$
q^{B}=\frac{\gamma(\beta-\Lambda \Gamma v)}{(1-\beta \gamma)(1-\Lambda \Gamma v \gamma)} .
$$

Note that in deriving the previous condition, I have not made use of the law of motion for rational bubbles given by (21), so (27) should hold for any deviation from fundamental pricing, whether rational or not. On the other hand, (21) must be used in order to derive the BGP relation between $q^{B}$ and $u$, with the latter denoting the corresponding (constant) ratio between the value of new bubbles and output:

$$
u=\left(1-\frac{1}{\Lambda \Gamma}\right) q^{B} .
$$

Next, I turn to a characterization of the model's balanced growth paths, i.e., the solutions to (27) and (28) satisfying $q^{B} \geq 0$ and $u \geq 0$.

\section{A. Bubbleless Balanced Growth Paths}

Consider first a "bubbleless" BGP, i.e., $q^{B}=u=0$, which is always a solution to (27) and (28). Imposing that condition implies

$$
\Lambda \Gamma v=\beta
$$

or, equivalently,

$$
r=(1+\rho)(1+g) v-1 \equiv r_{0} .
$$


Note that the real interest rate along a bubbleless BGP-henceforth denoted by $r_{0}$-is increasing in both $v$ and $g$. The reason is that an increase in either of those variables raises desired consumption by increasing the expected stream of future income for currently active individuals, for any given level of aggregate output. In order for the goods market to clear, an increase in the interest rate is called for.

When $v=1$ (i.e., no retirement), the real interest rate along the bubbleless BGP is given (approximately) by the discount rate plus the growth rate, i.e., $r_{0} \simeq \rho+g$, as in the standard representative agent model (with log utility).

Note also that a change in the expected lifetime, as indexed by $\gamma$, does not have an independent effect on $r_{0}$. The reason is that when $\Lambda \Gamma v=\beta$, a change in $\gamma$ scales in the same proportion the present value of consumption and that of income, leaving aggregate consumption unchanged and making an adjustment in the real rate unnecessary. 28

Finally, note for future reference that in the bubbleless BGP considered here, the real interest rate $r_{0}$ is lower than the growth rate $g$ (i.e., $\Lambda \Gamma>1$ ) if and only if $v<\beta$, a condition which is shown below to be critical for the existence of bubbles. ${ }^{29}$

\section{B. Balanced Growth Paths with Bubbles}

A BGP with a positive bubble corresponds to a solution to (27) and (28) satisfying $q^{B}>0$ and $u \geq 0$. Thus, the existence of a BGP with a positive bubble, $q^{B}>0$, requires that

$$
\Lambda \Gamma v<\beta
$$

or, equivalently, $r>r_{0}$. On the other hand, the nonnegativity constraint on newly created bubbles $u \geq 0$ requires

$$
\Lambda \Gamma \geq 1
$$

or, equivalently, $r \leq g$. The two previous conditions are satisfied if and only if

$$
v<\beta
$$

Note that the previous condition corresponds to the real interest rate being less than the growth rate in the bubbleless $B G P$ as shown above.

\footnotetext{
${ }^{28}$ The independence of the steady-state real interest rate from $\gamma$ is a consequence of the $\log$ utility specification assumed here. That property is not critical from the viewpoint of the present paper since there are other factors (the probability of retirement, in particular) that can drive the real interest rate to values consistent with the presence of bubbles.

${ }^{29}$ Note that the key role of retirement or, more generally, the anticipation of declining relative income in bringing about an interest rate lower than the growth rate was a central theme in Blanchard (1985) in a deterministic OLG model, though that paper did not discuss the possible existence of bubbles. On the other hand, in the classical models of bubbles using an OLG framework with two-period lives, the assumption of declining labor income, usually in the form of a lower endowment or no labor supply for the old, plays a key role in lowering the real interest rate and, hence, in generating the conditions for the existence of bubbles.
} 
One particular such bubbly BGP has no new bubbles introduced by new cohorts; i.e., $u=0$. Note that in that case, $\Lambda \Gamma=1$ or, equivalently, $r=g$. Thus, along that BGP, any existing bubble will be growing at the same rate as the economy, with the implied bubble size (relative to output) given by $q^{B}=\gamma(\beta-v) /((1-\beta \gamma)(1-v \gamma)) \equiv \bar{q}^{B}$.

The previous BGP is not the only bubbly BGP. If (29) is satisfied, there exists a continuum of bubbly BGPs indexed by $r \in\left(r_{0}, g\right]$. To see this, note that for any $r \in\left(r_{0}, g\right]$, equations (27) and (28) determine $q^{B}>0$ and $u \geq 0$ uniquely. It can be easily checked that $q^{B}$ is increasing in $r$, with $\lim _{r \rightarrow g} q^{B}=\bar{q}^{B}$, which establishes an upper bound to the size of the bubble-output ratio, given $\beta, \gamma$, and $v$. Note also that $\partial \bar{q}^{B} / \partial v<0$; i.e., the upper bound on the size of the bubble is decreasing in $v$ over the range $v \in[0, \beta]$ consistent with a bubbly BGP and converges to zero as $v \rightarrow \beta$.

Finally, note that along a bubbly BGP with bubble creation (i.e., with $u>0$ ), the size of any preexisting bubble will be shrinking over time relative to the size of the economy ( since $r<g$ ), with newly created bubbles filling up the gap so that the size of the aggregate bubble remains unchanged (relative to the size of the economy).

Summing up, one can distinguish two regions of the parameter space relevant for the possible existence of bubbly BGPs:

- $\beta \leq v \leq 1$. In this case, the BGP is unique and bubbleless and associated with a real interest rate given by $r_{0} \geq g$.

- $0<v<\beta$. In this case, multiple BGPs coexist. One of them is bubbleless, with $r=r_{0}<g$. In addition, there exists a continuum of bubbly BGPs, indexed by the real interest rate $r \in\left(r_{0}, g\right]$ and associated with a bubble size (relative to output) $q^{B} \in\left(0, \bar{q}^{B}\right]$, given by $(27) .{ }^{30}$

Figure 1 summarizes graphically the previous regions with their associated BGPs. ${ }^{31}$

\section{A Brief Detour: Bubbly Equilibria and Transversality Conditions}

Equilibria with bubbles on assets in positive net supply can be ruled out in an economy with an infinitely lived representative consumer. ${ }^{32}$ In that economy, any asset in positive net supply must necessarily be held by the representative consumer, implying

$$
\lim _{T \rightarrow \infty} E_{t}\left\{\Lambda_{t, t+T} A_{t+T}\right\} \geq \lim _{T \rightarrow \infty} E_{t}\left\{\Lambda_{t, t+T} Q_{t+T}^{B}\right\}
$$

\footnotetext{
${ }^{30}$ Any assessment of whether the $r \leq g$ condition is satisfied or not in an actual economy is likely to be controversial, but I think it is fair to say that there is no prima facie evidence against that condition, at least in the US economy. See, e.g., Blanchard (2019).

${ }^{31}$ Equations (27) and (28) can be used to derive an expression for $u$ as a function of $r \in\left(r_{0}, g\right)$. That function is equal to zero for the extreme values of $r$ 's domain, i.e., $r_{0}$ and $g$, and hump shaped for values in between. Accordingly, each $u$ is associated with two possible values of $r$ and, hence, $q$. It is thus not desirable to use $u$ to index the multiple BGPs.

${ }^{32}$ See, e.g., Santos and Woodford (1997) for a general analysis of the conditions under which rational bubbles can be ruled out in equilibrium.
} 


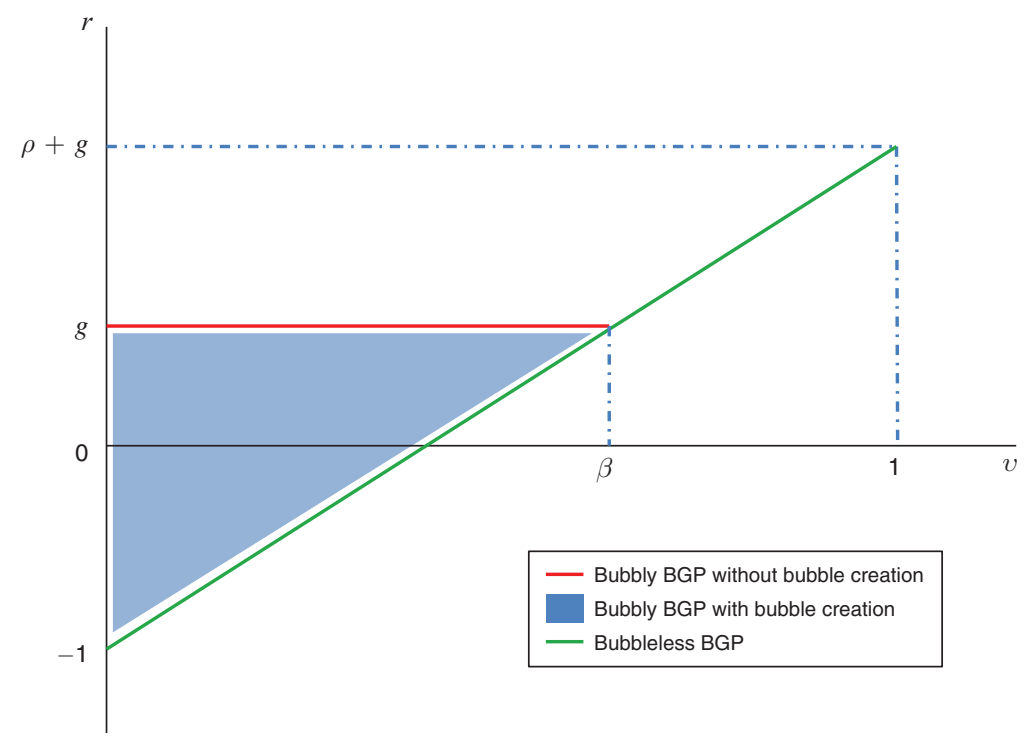

Figure 1. Balanced Growth Paths

On the other hand, the (rational) bubble component of any asset must satisfy

$$
\lim _{T \rightarrow \infty} E_{t}\left\{\Lambda_{t, t+T} Q_{t+T}^{B}\right\} \geq Q_{t}^{B}
$$

where the inequality allows for the possibility of new bubbles introduced after period $t$. It follows that $\lim _{T \rightarrow \infty} E_{t}\left\{\Lambda_{t, t+T} A_{t+T}\right\} \geq Q_{t}^{B}$. But the consumer's transversality condition requires that $\lim _{T \rightarrow \infty} E_{t}\left\{\Lambda_{t, t+T} A_{t+T}\right\}=0$. Free disposal rules out negative bubbles, so it must be the case that $Q_{t}^{B}=0$ for all $t$.

The previous reasoning cannot be applied to an overlapping generations economy like the one analyzed in the present paper. The reason is that it is no longer true in such an economy that the positive net supply of a bubbly asset must be held (asymptotically) by any individual agent since it can always be passed on to future cohorts (as it will, in equilibrium). In fact, it is easy to check that in the model above the individual transversality condition is satisfied along any BGP, bubbly or bubbleless. As shown in the Appendix, for an individual born in period $s \leq t$, it must be the case that along any BGP

$$
\lim _{T \rightarrow \infty} \gamma^{T} E_{t}\left\{\Lambda_{t, t+T} A_{t+T \mid s}\right\}=0,
$$

implying that the transversality condition is satisfied along any admissible BGP, including bubbly ones. It is straightforward to show that this will be the case along any equilibrium that remains in a neighborhood of a BGP, of the kind analyzed below. 


\section{Bubbles and Equilibrium Fluctuations}

Having characterized the BGPs of the OLG-NK economy, in the present section I shift the focus to the analysis of the equilibrium dynamics in a neighborhood of a given BGP. In particular, I am interested in determining the conditions under which bubble-driven aggregate fluctuations may emerge in equilibrium as well as the role that monetary policy may play in ruling out or stabilizing those fluctuations.

As in the standard analysis of the NK model with an infinitely lived representative agent, I restrict myself to equilibria that remain in a neighborhood of a BGP and approximate the equilibrium dynamics by means of the corresponding log-linearized equilibrium conditions. ${ }^{33}$ Throughout, I assume that the condition for a bubbly BGP is satisfied, i.e., $v \leq \beta$. I leave the analysis of the global equilibrium dynamicsincluding the possibility of switches between BGPs, the existence of a zero lower bound on interest rates, and other nonlinearities - to future research. Secondly, in analyzing the model's equilibrium, I ignore the existence of fundamental shocks and focus instead on the possibility of bubble-driven fluctuations. ${ }^{34}$

I start by deriving the log-linearized equilibrium conditions around a BGP. The resulting representation of the equilibrium dynamics takes a very simple form, involving only a few easily interpretable equations as shown next.

Let $\hat{c}_{t} \equiv \log \left(C_{t} / \Gamma^{t} \mathcal{C}\right)$ and $\hat{y}_{t} \equiv \log \left(Y_{t} / \Gamma^{t} \mathcal{Y}\right)$ denote log deviation of aggregate consumption and output from their value along a given BGP; the goods market clearing condition can be written as

$$
\hat{y}_{t}=\hat{c}_{t} \text {. }
$$

On the other hand, log-linearization of the aggregate consumption function (23), combined with (30), yields

$$
\hat{y}_{t}=(1-\beta \gamma)\left(\hat{q}_{t}^{B}+\hat{x}_{t}\right),
$$

where $\hat{q}_{t}^{B} \equiv q_{t}^{B}-q^{B}$, with $q_{t}^{B} \equiv Q_{t}^{B} /\left(\Gamma^{t} \mathcal{Y}\right)$ denoting the size of the aggregate bubble normalized by trend output and where $\hat{x}_{t} \equiv x_{t}-x$, with $x_{t} \equiv X_{t} /\left(\Gamma^{t} \mathcal{Y}\right)$ denoting aggregate fundamental wealth normalized by trend output, $x \equiv 1 /(1-\Lambda \Gamma v \gamma)$ being its value along the BGP.

Log-linearization of (24) around a BGP yields the following approximate expression for fundamental wealth, $\hat{x}_{t}$ :

$$
\hat{x}_{t}=\sum_{k=0}^{\infty}(\Lambda \Gamma v \gamma)^{k} E_{t}\left\{\hat{y}_{t+k}\right\}-\frac{\Lambda \Gamma v \gamma}{1-\Lambda \Gamma v \gamma} \sum_{k=0}^{\infty}(\Lambda \Gamma v \gamma)^{k} E_{t}\left\{\hat{r}_{t+k}\right\},
$$

with $\hat{r}_{t}=\hat{i}_{t}-E_{t}\left\{\pi_{t+1}\right\}$ denoting the real interest rate and $\hat{i}_{t}$ $\equiv \log \left[\left(1+i_{t}\right) /(1+r)\right]$ the nominal rate, all expressed in deviations from their

\footnotetext{
${ }^{33}$ See, e.g., Woodford (2003) and Galí (2015).

${ }^{34}$ As the analysis of the equilibrium dynamics below will make clear, in the absence of bubbles, the economy's behavior in response to fundamental shocks would involve no significant differences relative to that of the standard NK model.
} 
values along a zero inflation BGP. Note that $\hat{x}_{t}$ can be conveniently rewritten in recursive form as

$$
\begin{aligned}
\hat{x}_{t} & =\beta \gamma \Phi E_{t}\left\{\hat{x}_{t+1}\right\}+\hat{y}_{t}-\frac{\beta \gamma \Phi}{1-\beta \gamma \Phi} \hat{r}_{t} \\
& =\Phi E_{t}\left\{\hat{x}_{t+1}\right\}+\frac{1-\beta \gamma}{\beta \gamma} \hat{q}_{t}^{B}-\frac{\Phi}{1-\beta \gamma \Phi} \hat{r}_{t},
\end{aligned}
$$

where $\Phi \equiv \Lambda \Gamma v / \beta$.

As shown above, if $v \geq \beta$, the BGP is bubbleless and $\Phi=1$. On the other hand, if $v<\beta$, then $\Phi \in[v / \beta, 1]$, with its size inversely related to the size of the bubble along the BGP (or, equivalently, to the BGP real interest rate $r$ ).

Log-linearization of (21) around a BGP yields the equations describing fluctuations in the aggregate bubble:

$$
\begin{aligned}
& \hat{q}_{t}^{B}=\Lambda \Gamma E_{t}\left\{\hat{b}_{t+1}\right\}-q^{B} \hat{r}_{t}, \\
& \hat{q}_{t}^{B}=\hat{b}_{t}+\hat{u}_{t},
\end{aligned}
$$

where $\hat{u}_{t} \equiv u_{t}-u$, with $u_{t} \equiv U_{t} /\left(\Gamma^{t} \mathcal{Y}\right)$ denoting the size of the newly introduced bubble normalized by trend output and $u$ its value along the BGP. Note that (33) implies that the effect of a given interest rate change $\hat{r}_{t}$ on $\hat{q}_{t}^{B}$ is proportional to $q^{B}$, its value along the relevant BGP. The reason for this is that $\hat{q}_{t}^{B}$ measures the bubble as a fraction of aggregate output; hence, a given percent change in the (absolute) size of the bubble will translate into a larger change in $\hat{q}_{t}^{B}$ the larger is the initial bubble-output ratio. ${ }^{35}$

Together with the New Keynesian Phillips curve derived above and given by

$$
\pi_{t}=\beta \gamma \Phi E_{t}\left\{\pi_{t+1}\right\}+\kappa \hat{y}_{t},
$$

equations (31), (32), (33), and (34) provide a description of the nonpolicy block of the model's equilibrium in a neighborhood of a BGP, where the latter is defined by a pair $\left(q^{B}, \Lambda\right)$ satisfying the conditions derived in the previous section.

Note that a particular case of the OLG-NK model above is given by the bubbleless economy, with $q^{B}=0, \Phi=1$, and $\hat{q}_{t}^{B}=\hat{u}_{t}=0$ for all $t$. In that case, we can combine (31) and (32) to yield

$$
\hat{y}_{t}=E_{t}\left\{\hat{y}_{t+1}\right\}-\hat{r}_{t},
$$

which takes the same form as the so-called IS equation in the standard NK model. Thus, we see that the presence of finite horizons by itself does not help overcome the so-called "forward guidance puzzle" uncovered by a number of authors in the

\footnotetext{
${ }^{35}$ Accordingly, in a neighborhood of $q^{B}=0$, the change in $\hat{q}_{t}^{B}$ in response to a (first-order) change in $\hat{r}_{t}$ will be of second order.
} 
context of an infinitely lived representative agent model. ${ }^{36}$ Furthermore, note that (36) and (35) (after setting $\Phi=1$ ) provide a description of the nonpolicy block of the model which is isomorphic to that of a standard NK economy, albeit with a modified discount factor given by $\tilde{\beta}=\beta \gamma$. Accordingly, all the qualitative properties of alternative monetary policy rules found in that model will carry over to its OLG-NK extension considered here (e.g., the conditions for equilibrium uniqueness).

Next, I analyze the possibility of equilibrium bubble-driven fluctuations in the economy above. With little loss of generality and given the tight connection between inflation and the output gap implied by (35), much of the discussion below will focus on fluctuations in the latter variable, which simplifies the analysis considerably with little loss of generality. In order to build a better understanding of the role played by different assumptions in making bubble-driven fluctuations possible, I will proceed in steps and analyze the model's equilibrium under some limiting assumptions. First, I study the equilibrium under flexible prices, which provides a useful benchmark for the subsequent analysis. Secondly, I reintroduce sticky prices and analyze the properties of the model in the absence of bubble fluctuations in order to isolate the impact of finite lives and retirement on the model's properties. Thirdly, I study the possibility of bubble-driven fluctuations in the limiting case of a constant real interest rate. Finally, I endogenize the interest rate by assuming alternative monetary policy rules and study their implications on the possibility and properties of bubble-driven fluctuations that remain in a small neighborhood of the BGP. In all the cases considered, I take the BGP around which the economy fluctuates as exogenously given.

\section{A. Bubble Fluctuations under Flexible Prices}

As discussed above, in the equilibrium with flexible prices, the output gap is closed at all times, with output growing at a constant rate $g$, determined by the (exogenous) path of productivity. Accordingly, it follows from (31) that $\hat{x}_{t}=-\hat{q}_{t}^{B}$ for all $t$; i.e., changes in the size of the bubble crowd out fundamental wealth one-for-one, leaving aggregate demand unchanged. The necessary adjustment of private wealth is achieved through an appropriate change in the real interest rate. Combining the previous condition with (32), we obtain

$$
\hat{q}_{t}^{B}=\beta \gamma \Phi E_{t}\left\{\hat{q}_{t+1}^{B}\right\}+\frac{\beta \gamma \Phi}{1-\beta \gamma \Phi} \hat{r}_{t}^{n} .
$$

Iterating (37) forward, we obtain

$$
\hat{q}_{t}^{B}=\frac{\beta \gamma \Phi}{1-\beta \gamma \Phi} \sum_{k=0}^{\infty}(\beta \gamma \Phi)^{k} E_{t}\left\{\hat{r}_{t+k}^{n}\right\},
$$

which makes clear that any variation in the size of the aggregate bubble must be met in equilibrium with an appropriate change in current and/or expected future real

\footnotetext{
${ }^{36}$ See, e.g., Carlstrom, Fuerst, and Paustian (2015); Del Negro, Giannoni, and Patterson (2015); and McKay, Nakamura, and Steinsson (2016) for a discussion of the forward guidance puzzle.
} 
interest rates in order to neutralize the bubble's impact on aggregate demand and leave the output gap unchanged.

Equations (33), (34), and (37) jointly describe the equilibrium behavior of the aggregate bubble and the natural rate, $\hat{r}_{t}^{n}$. Combining the three equations, we can derive the following equilibrium condition for the aggregate bubble:

$$
\hat{q}_{t}^{B}=\Psi E_{t}\left\{\hat{q}_{t+1}^{B}\right\}-\Upsilon E_{t}\left\{\hat{u}_{t+1}\right\},
$$

where $\Psi \equiv \Phi\left[1+(\Lambda \Gamma-1) \frac{1-\beta \gamma}{1-\beta \gamma \Phi}\right]>0$ and $\Upsilon \equiv \frac{\Phi \Lambda \Gamma(1-\beta \gamma)}{1-\beta \gamma \Phi}>0$.

Note that $\hat{q}_{t}^{B}=\hat{u}_{t}=0$ for all $t$ is always a solution to (38) and hence a possible equilibrium in which the size of both the aggregate bubble and of newly created bubbles grows at a constant rate along the BGP. But (38) may have other nonexplosive solutions, associated with stationary bubble fluctuations. In order to analyze that possibility, I distinguish two generic cases, depending on whether $\Psi$ is smaller or larger than one. Later, I discuss the determinants of the size of $\Psi$. by 37

If $\Psi<1$, the only stationary solution to (38) is the forward-stable one, given

$$
\hat{q}_{t}^{B}=-\Upsilon \sum_{k=1}^{\infty} \Psi^{k-1} E_{t}\left\{\hat{u}_{t+k}\right\}
$$

One particular class of solutions to (39) involves no predictable deviations of newly created bubbles from their BGP value (i.e., $E_{t}\left\{\hat{u}_{t+k}\right\}=0$, for $k=1,2, \ldots$ and all $t$ ). In that case, the size of the aggregate bubble remains constant (i.e., $\hat{q}_{t}^{B}=0$ for all $t$ ), with any (unanticipated) change in $\hat{u}_{t}$ crowding out one-for-one preexisting

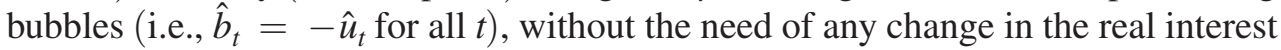
rate as implied by (37). ${ }^{38}$ More generally, there are (infinitely) many other solutions to (39) that involve forecastable variations in the size of future new bubbles and, as a result, changes in the current aggregate bubble as well. A property of those solutions is that expectations of larger newly created bubbles in the future (i.e., $E_{t}\left\{\hat{u}_{t+k}\right\}>0$, for $k=1,2, \ldots$ ) are associated with a smaller size of the current bubble as implied by (39). That crowding out is needed in order to guarantee a nonexplosive path for the aggregate bubble, given the required change in the interest rate consistent with an unchanged output (see further discussion below). This kind of bubble fluctuations, associated with anticipated deviations in the size of newly created bubbles, was the focus of the analysis in Miao, Shen, and Wang (2019) in the context of a two-period OLG model, though their analysis was restricted to the case of newly created bubbles following an exogenous AR(1) process.

\footnotetext{
${ }^{37}$ In the case of $\Psi=1$, I further assume $\sum_{k=1}^{\infty} E_{t}\left\{\hat{u}_{t+k}\right\}<+\infty$.

${ }^{38}$ Note that after a (temporary) shock to $\hat{u}_{t}$, all variables return to their BGP values after one period, with $\hat{b}_{t+1}=0$, which is consistent with $\hat{q}_{t}^{B}=\hat{r}_{t}=0$ in (33).
} 
When $\Psi>1$, on the other hand, any eventual stationary bubble fluctuations are described by the backward-stable solution to (38), given by

$$
\hat{q}_{t}^{B}=\frac{1}{\Psi} \hat{q}_{t-1}^{B}+\frac{\Upsilon}{\Psi} E_{t-1}\left\{\hat{u}_{t}\right\}+\varepsilon_{t},
$$

where $\varepsilon_{t} \equiv q_{t}^{B}-E_{t-1}\left\{q_{t}^{B}\right\}=\left(b_{t}-E_{t-1}\left\{b_{t}\right\}\right)+\left(u_{t}-E_{t-1}\left\{u_{t}\right\}\right)$ is the bubble innovation, which in turn is the sum of the unanticipated changes in preexisting and newly created bubbles. Note that in this case, both types of innovations generate a persistent response of the aggregate bubble of the same sign. Furthermore, and in contrast with the $\Psi<1$ case considered above, such persistent aggregate bubble fluctuations may arise even if newly created bubbles are constant or display unpredictable variations. In that case, (40) simplifies to

$$
\hat{q}_{t}^{B}=\frac{1}{\Psi} \hat{q}_{t-1}^{B}+\varepsilon_{t}
$$

with the (natural) interest rate changing in proportion to the size of the aggregate bubble and given by

$$
\hat{r}_{t}^{n}=\left(1-\frac{\beta \gamma \Phi}{\Psi}\right)\left(\frac{1-\beta \gamma \Phi}{\beta \gamma \Phi}\right) \hat{q}_{t}^{B}
$$

Changes in the aggregate bubble in this case are the result of (self-fulfilling) waves of optimism or pessimism among investors regarding the future size of existing bubbles. I interpret this type of bubble fluctuations (rather than the one associated with (39)) as better capturing traditional accounts of the driving forces behind speculative bubble episodes, which usually involve investors' willingness to pay a "high" price for an asset on the basis of further anticipated increases in the price of the same asset (as opposed to an anticipated decrease in the size of future newly created bubbles).

Next, I show that the size of $\Psi$ and, hence, the properties of any eventual stationary bubble fluctuations in the equilibrium above depend critically on $q^{B}$, the size of the bubble-output ratio along the BGP. To see this, consider first the case of $v \geq \beta$, which implies $q^{B}=u=0$ and $\Psi=\Lambda \Gamma=\beta / v \leq 1$. Thus, any stationary bubble fluctuations should satisfy (39). But the nonnegativity constraints on $q_{t}^{B}$ and $u_{t}$ imply that the only admissible solution is $q_{t}^{B}=u_{t}=0$ for all $t$, thus ruling out altogether bubble fluctuations in that case.

Next, consider the case of $v<\beta$, which is consistent with a continuum of BGP bubble-output ratios. Let me examine first the two limiting cases, $q^{B}=0$ and $q^{B}=\bar{q}^{B}$. If $q^{B}=0$, then $\Psi=\beta / v>1$, so the condition for stationary bubble fluctuations given by (40) is satisfied. On the other hand, if $q^{B}=\bar{q}^{B}$, then $\Psi=v / \beta<1$ so that only bubble fluctuations of the type given by (39) may exist. It is easy to check that $\Psi$ is continuous and differentiable in $q^{B}$, with $d \Psi / d q^{B}<0$. It follows that there always exists a threshold bubble-output ratio $q_{*}^{B}$ such that $\Psi>1$ if $q^{B}<q_{*}^{B}$ and, conversely, $\Psi \leq 1$ if $q^{B} \geq q_{*}^{B}$. Thus, under the assumption that $v<\beta$, the possibility of stationary bubble fluctuations always arises, with the nature of those fluctuations depending critically on the size of $q^{B}$. 
To gain some intuition on the role of $q^{B}$ in determining the nature of any eventual stationary bubble fluctuations, consider a perfect foresight path followed by the aggregate bubble starting from period $t$ on as implied by (33):

$$
\hat{q}_{t+1}^{B}=(\Lambda \Gamma)^{-1} \hat{q}_{t}^{B}+(\Lambda \Gamma)^{-1} q^{B} \hat{r}_{t}+\hat{u}_{t+1},
$$

for $k=0,1,2, \ldots$ and assuming $\lim _{T \rightarrow \infty} \hat{u}_{t+T}=0$. Given the discussion above, I focus on the case of $v<\beta$, which allows for a nonzero bubble ratio $q^{B}$ along the BGP. As discussed in the BGP analysis, $r$, and hence, $(\Lambda \Gamma)^{-1}$ are increasing in $q^{B}$, with $\lim _{q^{B} \rightarrow \bar{q}^{B}} \Lambda \Gamma=1$. Also, recall that the interest rate comoves positively with the bubble in a way consistent with a constant output gap in equilibrium. Thus, if $q^{B}$ and $r$ are "sufficiently small," the low inherent persistence of the bubble (measured by $\left.(\Lambda \Gamma)^{-1}\right)$, combined with the limited impact on the bubble of the accompanying changes in the interest rate, are sufficient to guarantee that $\lim _{T \rightarrow+\infty} \hat{q}_{t+T}^{B}=0$ given any initial value $\hat{q}_{t}^{B}$, thus allowing for stationary fluctuations. By contrast, if $q^{B}$ and $r$ are "sufficiently large," the high inherent persistence of the bubble, combined with its strong sensitivity to interest rate changes, imply that $\hat{q}_{t}^{B}=\hat{r}_{t}=0$ is the only nonexplosive solution if $\hat{u}_{t+k}=0$, for $k=1,2, \ldots$ On the other hand, "news" at time $t$ of, say, an increase in the size of a future new bubble (e.g., $\hat{u}_{t+k}>0$, for some $k>0$ ) requires an immediate fall in $\hat{q}_{t}^{B}$ (determined by (39)) to guarantee convergence back to the BGP once the anticipated larger new bubble is realized.

The assumption of flexible prices maintained throughout the present section has two extreme (and arguably unrealistic) implications. Firstly, and as noted above, when combined with the absence of "fundamental" shocks, it leads to a constant output gap, even in the presence of bubble fluctuations. Secondly, it implies that real variables are determined in equilibrium independently of monetary policy. ${ }^{39}$ In the remainder of the paper, I reintroduce sticky prices and analyze the equilibrium of the resulting OLG-NK model, with a focus on the interaction between monetary policy and bubble and output fluctuations.

\section{B. Bubble-Driven Fluctuations in the Equilibrium with Sticky Prices}

Next, I turn to the analysis of the OLG-NK economy with sticky prices. Once the assumption of flexible prices is relaxed, output is no longer being determined by supply side factors and will be affected by the impact of bubble fluctuations on aggregate demand. In addition, monetary policy is no longer neutral. Accordingly, and in order to close the model, equations (31) through (35) - the equilibrium conditions describing the behavior of the private sector-must be complemented with a monetary policy rule. In order to

\footnotetext{
${ }^{39}$ Though not the focus of my analysis, it is clear that monetary policy may still have an impact on nominal variables under flexible prices. Given the path of $\hat{r}_{t}^{n}$, one can combine the Fisherian equation $\hat{i}_{t}=\hat{r}_{t}^{n}+\pi_{t}$ with a suitable interest rate rule to determine the equilibrium path of inflation and other nominal variables. See, e.g., chapter 2 in Gali (2015) for an analysis of the determination of nominal variables in a model with flexible prices and a real rate independent of monetary policy, like the present one.
} 
keep things as simple as possible, henceforth I assume the following interest rate rule:

$$
\hat{i}_{t}=E_{t}\left\{\pi_{t+1}\right\}+\phi_{y} \hat{y}_{t}+\phi_{q} \hat{q}_{t}^{B}
$$

where $\hat{i}_{t} \equiv \log \left[\left(1+i_{t}\right) /(1+r)\right]$.

Note that the previous rule combines the usual stabilization motive (parameterized by $\phi_{y} \geq 0$ ) with a possible desire to "lean against the bubble" (LAB, for short), parameterized by $\phi_{q} \geq 0$. Throughout, I assume the central bank takes as given the BGP around which the economy fluctuates (and, hence, $r$ and $q^{B}$ ). ${ }^{40}$ Given the simple relation between inflation and the output gap implied by (35), the assumption of a rule like (43), which can be written in terms of the real rate and involves no "direct" response to inflation, makes it possible to solve for the model's real variables without any reference to (35), thus simplifying the analysis considerably and allowing me to obtain some analytical results. ${ }^{41}$

Equations (31), (32), (33), (34), and (43) describe the equilibrium behavior of $\hat{y}_{t}, \hat{r}_{t}, \hat{x}_{t}, \hat{q}_{t}^{B}, \hat{b}_{t}$, and $\hat{u}_{t}$ in a neighborhood of a given BGP. A quick glance at those equations makes clear that an outcome with all of them equal to zero for all $t$ always constitutes a solution to that system of equations. In other words, the perfect foresight BGP itself is always an equilibrium. This should not be surprising, given that fundamental shocks have been deliberately assumed away. The question of interest, however, is whether that outcome is the only possible equilibrium and, more precisely, whether other equilibria exist involving aggregate fluctuations that are bubble driven.

In order to isolate such bubble-driven fluctuations, however, I also need to rule out equilibria involving expectations-driven fluctuations unrelated to the existence of bubble fluctuations, i.e., conventional sunspot fluctuations. With that objective in mind, next I analyze the conditions for a unique equilibrium in the OLG-NK model without bubble fluctuations.

A Brief Detour: Equilibrium in the Absence of Bubble Fluctuations.-Consider the economy described by (31), (32), (33), (34), and (43) on which we impose the assumption of no bubble fluctuations, i.e., $\hat{q}_{t}^{B}=\hat{u}_{t}=0$ for all $t$. A particular case of such an environment corresponds to the OLG-NK model without bubbles, i.e., with $q^{B}=0$ as well as $q_{t}^{B}=u_{t}=0$ for all $t$. Combining (31), (32), and (43) after imposing $\hat{q}_{t}^{B}=\hat{u}_{t}=0$ for all $t$, we obtain the difference equation

$$
\hat{y}_{t}=\Xi E_{t}\left\{\hat{y}_{t+1}\right\}
$$

\footnotetext{
${ }^{40}$ The assumption of policy invariance of $r$ and $q^{B}$ is not an obvious one in the present model, where a multiplicity of real interest rates and bubble-output ratios may be consistent with a BGP if certain conditions are met. This may be viewed as a shortcoming of the present approach that should deserve attention in future research.

${ }^{41}$ Note that the "divine coincidence" property implies that stabilizing the output gap is equivalent to stabilizing inflation and vice versa.
} 
where $\Xi \equiv \frac{\Phi(1-\beta \gamma \Phi)}{1-\beta \gamma \Phi+\Phi(1-\beta \gamma) \phi_{y}}$, with $\Phi \in[v / \beta, 1]$. Thus, in the absence of bubble fluctuations, there is unique stationary equilibrium given by $\hat{y}_{t}=0$ for all $t$, if and only if $\Xi \leq 1$ or, equivalently,

$$
\phi_{y} \geq-\frac{(1-\Phi)(1-\beta \gamma \Phi)}{\Phi(1-\beta \gamma)} \equiv \phi_{y}^{0} .
$$

Note that in the case of a bubbleless BGP (and, hence, in the absence of bubbles altogether), $\Phi=1$ and $\phi_{y}^{0}=0$, so the previous condition takes the simple form $\phi_{y} \geq 0$. The standard NK model is a particular case of bubbleless BGP, so $\phi_{y} \geq 0$ is the relevant condition for that model. ${ }^{42}$ On the other hand, in the case of a bubbly BGP (i.e., $q^{B}>0$ ), $\phi_{y}^{0}<0$ and, hence, the condition for a unique equilibrium is relaxed somewhat, allowing for (slightly) negative values for $\phi_{y}$.

Most importantly for the analysis herein, it follows from the previous finding and the maintained assumption of no fundamental shocks that any stationary fluctuations in output in the OLG-NK model when $\phi_{y} \geq \phi_{y}^{0}$ must be bubble driven.

Next, I turn to the analysis of such bubble-driven fluctuations, under the maintained assumption that $\phi_{y} \geq \phi_{y}^{0}$ (which rules out stationary sunspot fluctuations unrelated to variations in the size of the aggregate bubble). For simplicity, henceforth I restrict the analysis to fluctuations in which the size of newly created bubbles is unforecastable (i.e., $E_{t}\left\{\hat{u}_{t+k}\right\}=0$ for $k=1,2, \ldots$ and all $t$ ). There are several reasons for doing so. Firstly, allowing for unrestricted expectations on the size of future new bubbles generates an embarrassment of riches in terms of possible outcomes, making any rigorous discussion long and tedious. ${ }^{43}$ Secondly, and as discussed above in the context of the flexible price version of the model, the assumption of unforecastable new bubbles narrows the range of possible outcomes by ruling out stationary equilibria in which anticipated future newly created bubbles crowd out the current aggregate bubble, generating a negative comovement between the two. That mechanism, while theoretically possible, seems at odds with anything observed in reality. I focus instead on bubble fluctuations that result from revisions in expectations about the future value of current bubbles (new or preexisting), which in turn lead to adjustments on the value of those bubbles. Those fluctuations capture better, in my opinion, actual speculative bubble episodes, with mood swings leading investors to pay a higher (lower) price for an asset purely on the basis of expectations of further increases (decreases) in the price of the same asset, despite the absence of any news regarding its fundamental payoffs or the size of future new bubbles on different assets.

Note, however, that the assumption of newly created bubbles still plays two important roles even if one assumes their variations to be unforecastable.

\footnotetext{
${ }^{42}$ Note that in the standard NK model with an infinitely lived representative agent, $\Phi=1$ and $\gamma=1$, thus implying $\Xi \equiv 1 /\left(1+\phi_{y}\right)$. Accordingly, $\phi_{y} \geq 0$ is necessary and sufficient for a unique solution. It can be easily checked that given the assumed rule, the previous condition implies an (eventual) increase in the nominal rate of more than one-for-one in response to a change in inflation, i.e., the familiar Taylor principle.

${ }^{43}$ Miao, Shen, and Wang (2019) assume an $\operatorname{AR}(1)$ process for $\left\{u_{t}\right\}$, thus restricting somewhat the range of possible equilibria. A similar assumption is often made in the context of business cycle models, albeit applied to an exogenous driving process. But given that $\left\{u_{t}\right\}$ is an endogenous variable, it is not clear how one should interpret such a parametric assumption.
} 
Firstly, their presence in a BGP (i.e., $u>0$ ) implies that $r<g$ and $q^{B}<\bar{q}^{B}$, which opens the door to stationary bubble-driven fluctuations that would not otherwise be possible, as I show below. Secondly, newly created bubbles make it possible to rekindle an aggregate bubble after the collapse of the latter (see the stochastic bubble example below as an illustration).

The Special Case of a Constant Real Interest Rate.- In order to build some intuition on the mechanisms through which aggregate bubble fluctuations are transmitted to the real economy and the role of monetary policy in shaping those fluctuations, I start by analyzing the special case in which the central bank is assumed to maintain a constant real interest rate, regardless of the path of output and/or the aggregate bubble. ${ }^{44}$ As discussed above, I assume newly created bubbles are unforecastable (i.e., $E_{t}\left\{\hat{u}_{t+k}\right\}=0$, for $k=1,2, \ldots$, and for all $t$ ). Under the previous assumptions, the equilibrium dynamics can be summarized by the following two equations:

$$
\begin{aligned}
& \hat{y}_{t}=\Phi E_{t}\left\{\hat{y}_{t+1}\right\}+\frac{(1-\beta \gamma)(1-v \gamma)}{\beta \gamma} \hat{q}_{t}^{B}, \\
& \hat{q}_{t}^{B}=\Lambda \Gamma E_{t}\left\{\hat{q}_{t+1}^{B}\right\},
\end{aligned}
$$

which can be derived by combining (31), (32), (33), and (34) under the assumption that $\hat{r}_{t}=0$ for all $t$.

Note that an outcome involving no fluctuations (i.e., $\hat{y}_{t}=\hat{q}_{t}^{B}=0$, for all $t$ ) always constitutes an equilibrium. That outcome is also the only stationary equilibrium if $v \geq \beta$, for in that case $\Lambda \Gamma \leq 1$, which is inconsistent with stationary fluctuations in the aggregate bubble. The same is true if $v<\beta$ and $q^{B}=\bar{q}^{B}$. On the other hand, under the assumption that $v<\beta$ and $q^{B}<\bar{q}^{B}$, we have $\Lambda \Gamma>1$, so the bubble may display stationary fluctuations consistent with (45) and described by the $\operatorname{AR}(1)$ process

$$
\hat{q}_{t}^{B}=(\Lambda \Gamma)^{-1} \hat{q}_{t-1}^{B}+\varepsilon_{t},
$$

where $\varepsilon_{t} \equiv \hat{b}_{t}-E_{t-1}\left\{\hat{b}_{t}\right\}+\hat{u}_{t}$, a martingale difference process, is the innovation in the aggregate bubble (the "bubble shock," for short). Note that the persistence of the bubble, measured by $(\Lambda \Gamma)^{-1}$, lies between $v / \beta$ and 1 , so it is likely to be very high for any plausible calibration. Equilibrium output can then be solved for using (44):

$$
\hat{y}_{t}=\Omega \hat{q}_{t}^{B},
$$

where $\Omega \equiv(1-\beta \gamma)(1-v \gamma) /(\gamma(\beta-v))>0$ can be interpreted as the bubble multiplier under a constant interest rate. Equivalently,

$$
\hat{y}_{t}=(\Lambda \Gamma)^{-1} \hat{y}_{t}+\Omega \varepsilon_{t} .
$$

\footnotetext{
${ }^{44}$ Woodford (2011) makes an identical assumption as a preliminary step in his analysis of the size of government spending multipliers.
} 
There are several channels at work in determining that impact of the bubble on output. Firstly, there is a direct effect of a larger bubble on consumption, of size $1-\beta \gamma$, as implied by (31). Secondly, there is a static multiplier channel, of size $(1-\beta \gamma)^{2} /(\beta \gamma)$, associated with the higher consumption induced by the contemporaneous increase in output, as implied by combining (31) and (32), while keeping future output constant. And, finally, a dynamic multiplier channel, of size $v(1-\beta \gamma)^{2} /(\beta \gamma(\beta-v))$, resulting from the higher consumption induced by the anticipation of higher future output, with the latter being in turn a consequence of the persistent response of the bubble.

Given the natural upper bound of unity for $\beta$, a lower bound on the (constant interest rate) bubble multiplier $\Omega$ is $(1-\gamma)(1-v \gamma) /(\gamma(1-v))$, which has an order of magnitude of 0.01 , a relatively small value, for any reasonable values for $\gamma$ and $v$. On the other hand, the size of that multiplier becomes unbounded as $(\beta-v) \rightarrow 0$. As one approaches that limiting case, and due to the high persistence of the bubble, any initial increase in output resulting from a bubble shock generates an expected discounted value of future income equal to $(1-\beta \gamma)^{-1} \hat{y}_{t}$ and thus an "effective" marginal propensity to consume out of current income of unity, leading to an infinite feedback effect. Thus, we see that the range of possible values for the bubble multiplier under the constant interest rate assumption considered here is very wide, potentially including very large values. This is the case even though the marginal propensity to consume out of wealth $(1-\beta \gamma)$-which determines only the direct, partial equilibrium effect—is likely to be small.

Of course, with an endogenous real rate, the assumed monetary policy rule becomes an important factor in determining the effective bubble multiplier. Next, I relax the assumption of a constant interest rate and analyze the equilibrium dynamics for the general case.

Bubble-Driven Fluctuations with an Endogenous Interest Rate.-Next, I study the possibility of bubble-driven fluctuations when the real interest rate varies endogenously, according to policy rule (43). It is useful to consider the cases of fluctuations around a bubbleless BGP $\left(q^{B}=0\right)$ and a bubbly BGP $0<q^{B} \leq \bar{q}^{B}$ separately. Again, I maintain the assumption of constant or unpredictable newly created bubbles.

Fluctuations near a Bubbleless BGP.- The particular case of bubble-driven fluctuations near the bubbleless BGP $\left(q^{B}=u=0\right)$ is of special interest for at least two reasons. Firstly, the equilibrium dynamics are simplified by the fact that to a first-order approximation, there is no impact of monetary policy on the bubble ratio itself. On the other hand, bubble fluctuations may still affect aggregate demand and output through their impact on monetary policy if $\phi_{q}>0$. Secondly, this case may be viewed as a reasonable representation of recurrent episodes of a rise and subsequent collapse of aggregate bubbles similar to those that are apparent in actual economies, with the no bubble state being a "natural" resting point. ${ }^{45}$

${ }^{45}$ The dot-com bubble of the late 1990s and the housing bubbles of the 2000s are an example of such episodes. 
Near the bubbleless BGP, the bubble evolves "autonomously" according to the process

$$
q_{t}^{B}=\frac{\beta}{v} E_{t}\left\{q_{t+1}^{B}\right\}
$$

where $q_{t}^{B} \geq 0$ and $u_{t} \geq 0$, for all $t .^{46}$ The assumption $q^{B}=0$ made here implies that interest rate changes do not affect (up to a first-order approximation) the expected evolution of the bubble-output ratio $q_{t}^{B}$. If $v \geq \beta$, the only stationary solution to (46) is $q_{t}^{B}=0$ for all $t$, thus ruling out stationary bubble fluctuations and, assuming $\phi_{y} \geq 0$, output fluctuations as well. ${ }^{47}$ By contrast, if $v<\beta$, other stationary solutions to (46) exist, of the form

$$
q_{t}^{B}=\frac{v}{\beta} q_{t-1}^{B}+\varepsilon_{t},
$$

where $\varepsilon_{t} \equiv b_{t}-E_{t-1}\left\{b_{t}\right\}+u_{t}-E\left\{u_{t}\right\}$ is the aggregate bubble innovation, a martingale difference process. Note that the aggregate bubble will remain "small" (thus justifying the approximation around $q^{B}=0$ ) as long as $E\left\{u_{t}\right\} \gtrsim 0 .{ }^{48}$ On the other hand, we can combine (31), (32), and (43) to obtain the following equilibrium condition for the output gap:

$$
\hat{y}_{t}=\frac{1}{1+\phi_{y}} E_{t}\left\{\hat{y}_{t+1}\right\}+\frac{1}{1+\phi_{y}}\left(\frac{1-\beta \gamma}{\beta \gamma}-\phi_{q}\right) q_{t}^{B}-\frac{1-\beta \gamma}{1+\phi_{y}} E_{t}\left\{q_{t+1}^{B}\right\} .
$$

Finally, one can combine (46) and (48) to derive an expression for the output gap as a function of the aggregate bubble:

$$
\hat{y}_{t}=\left(\frac{(1-\beta \gamma)(1-v \gamma)}{\beta \gamma}-\phi_{q}\right)\left(\frac{1}{1-\frac{v}{\beta}+\phi_{y}}\right) q_{t}^{B} .
$$

Under the assumption that $\phi_{q} \leq(1-\beta \gamma)(1-v \gamma) /(\beta \gamma)$-i.e., a not too aggressive "lean-against-the-bubble" policy (LAB policy, henceforth) - the output gap comoves positively with the aggregate bubble, arguably the more realistic case. Even though in that environment the central bank has no influence on the bubble itself, it has two options in order to stabilize the impact of bubble fluctuations on aggregate demand and output. First, it can choose a value of $\phi_{q}$ close to $(1-\beta \gamma)(1-v \gamma) /(\beta \gamma)$, which can be interpreted as a "surgical" LAB policy. In that case, the strength of the response is calibrated so that the interest rate matches exactly the natural rate, which from (42) can be shown to be given by $\hat{r}_{t}^{n}=((1-\beta \gamma)(1-v \gamma) /(\beta \gamma)) q_{t}^{B}$ under the present assumptions. Note, however, that changes in $\phi_{q}$ have a nonmonotonic impact on the response of output to bubble fluctuations. In particular, if $\phi_{q}>(1-\beta \gamma)(1-v \gamma) /(\beta \gamma)$, the strong interest rate response to variations in the size of the aggregate bubble will more than offset the impact of the latter on

\footnotetext{
${ }^{46}$ In order to derive (46), I have made use of (33) and the fact that $\Lambda \Gamma v=\beta$ when $q^{B}=0$.

${ }^{47}$ Recall from the analysis above that $\phi_{y} \geq 0$ is a sufficient condition to rule out stationary output fluctuations in the absence of bubble fluctuations.

${ }^{48}$ It is easy to check that $\lim _{T \rightarrow \infty} E_{t}\left\{q_{t+T}^{B}\right\}=\beta E\left\{u_{t}\right\} /(\beta-v)$, which can be arbitrarily small as the unconditional mean $E\left\{u_{t}\right\}$ approaches zero.
} 
the economy, reversing its sign and generating a negative comovement between the aggregate bubble and the output gap resulting from the central bank's "overreaction." Alternatively, and perhaps more robustly, the central bank may "neglect" bubble developments and focus on stabilizing the output gap (or, equivalently, inflation, given (35)) by choosing an arbitrarily large $\phi_{y}$ coefficient.

The previous environment allows for the possibility of recurrent booms and busts driven by a "stochastic bubble" of the kind proposed in Blanchard (1979), which evolves according to the process

$$
q_{t}^{B}= \begin{cases}\frac{v}{\beta \delta} q_{t-1}^{B}+u_{t} & \text { with probability } \delta \\ u_{t} & \text { with probability } 1-\delta,\end{cases}
$$

where $\left\{u_{t}\right\}$ is a white noise process with positive support and unconditional mean $E\left\{u_{t}\right\} \gtrsim 0$. It is easy to check that the previous process satisfies (46) as well as the nonnegativity condition. The economy's equilibrium, described by (49) and (50), will display recurrent output booms, driven by rapid bubble growth, followed by eventual (though unpredictable) collapses, before being rekindled again by new bubbles. 49

Fluctuations around a Bubbly BGP.-Next, I consider the possibility of bubble-driven fluctuations around a BGP with a positive bubble, i.e., with $q^{B} \in$ $\left(0, \bar{q}^{B}\right]$ and $r \in\left(r_{0}, g\right]$. Accordingly, I maintain the assumption that $v<\beta$, which is necessary for such a bubbly BGP to exist. A key difference here relative to the case of fluctuations near a bubbleless BGP analyzed above is that the evolution of the bubble-output ratio $q_{t}^{B}$ is affected (to a first order) by the real interest rate, as implied by (33). This complicates the analysis somewhat, but some progress can be made in understanding the key elements at play by looking at some special cases.

Consider first the case of no direct output stabilization motive (i.e., $\phi_{y}=0$ ) while allowing for an active LAB policy (i.e., $\phi_{q} \geq 0$ ). In that case, we can combine equations (33) and (43) to obtain the following simple equilibrium equation for the aggregate bubble, in deviations from its value at the BGP:

$$
\hat{q}_{t}^{B}=\frac{\Lambda \Gamma}{1+q^{B} \phi_{q}} E_{t}\left\{\hat{q}_{t+1}^{B}\right\} .
$$

The stationary solution to (51) is unique and given by $\hat{q}_{t}^{B}=0$ for all $t$ if and only if

$$
\phi_{q}>\frac{\Lambda \Gamma-1}{q^{B}} \equiv \phi_{q}^{*} .
$$

If the previous condition is satisfied, it follows from (31) and (32) that $\hat{y}_{t}=0$ for all $t$. In words: if $q^{B}>0$, the central bank can always rule out bubble fluctuations (and the associated output fluctuations) by committing to a sufficiently aggressive LAB policy. How strong that response must be is given by the

\footnotetext{
${ }^{49}$ The statement assumes $\phi_{q} \in[0,(1-\beta \gamma)(1-\beta v) /(\beta \gamma)]$. See discussion above.
} 
threshold $\phi_{q}^{*} \geq 0$, which in turn depends crucially on $q^{B}$ since the latter determines the impact of interest changes on the size of the bubble-output ratio. ${ }^{50}$ As $q^{B} \rightarrow \bar{q}^{B}$, we have $\Lambda \Gamma \rightarrow 1$ and, hence, $\phi_{q}^{*} \rightarrow 0$. Thus, if the size of the bubble-output ratio along the BGP is "large" (i.e., close to its upper bound), a rule that commits to responding even weakly to fluctuations in $\hat{q}_{t}^{B}$ were they to arise will suffice to rule out such fluctuations. At the other extreme, as $q^{B} \rightarrow 0$ (and $\Lambda \Gamma \rightarrow \beta / v>1$ ), we have $\phi_{q}^{*} \rightarrow+\infty$; i.e., no systematic policy response to the bubble, no matter how aggressive, will be effective at ruling out bubble fluctuations. More generally, note that the threshold $\phi_{q}^{*}$ is decreasing in $q^{B}$; i.e., the required policy response is larger the smaller is the bubble-output ratio along the BGP. See Figure 2.

On the other hand, if $0 \leq \phi_{q}<\phi_{q}^{*}$, there exist equilibria with stationary bubble fluctuations satisfying the stationary $\mathrm{AR}(1)$ process

$$
\hat{q}_{t}^{B}=\left(\frac{1+q^{B} \phi_{q}}{\Lambda \Gamma}\right) \hat{q}_{t-1}^{B}+\varepsilon_{t},
$$

where $\varepsilon_{t} \equiv b_{t}-E_{t-1}\left\{b_{t}\right\}+\hat{u}_{t}$. Under the assumption that the bubble innovation process $\left\{\varepsilon_{t}\right\}$ is independent of monetary policy, a (local) increase in $\phi_{q}$ will result in an increase in the volatility of the aggregate bubble. That property is a consequence of the equilibrium requirement that the anticipated return on the bubble equals the interest rate. The endogenous rise in the latter called for by the LAB policy will thus "amplify" the impact over time on the size of the bubble of an initial bubble shock. This is consistent with the findings emphasized in Galí (2014) in the context of a two-period OLG model. ${ }^{51}$

More generally, if $\phi_{y} \geq 0$, one can combine (31), (32), and (43) to obtain, after some algebra, the following representation of the joint equilibrium dynamics for the output gap and the aggregate bubble:

$$
\left[\begin{array}{l}
\hat{y}_{t} \\
\hat{q}_{t}^{B}
\end{array}\right]=\left[\begin{array}{ll}
A_{11} & A_{12} \\
A_{21} & A_{22}
\end{array}\right]\left[\begin{array}{l}
E_{t}\left\{\hat{y}_{t+1}\right\} \\
E_{t}\left\{\hat{q}_{t+1}^{B}\right\}
\end{array}\right],
$$

where $A_{11} \equiv \Theta \Phi, \quad A_{12} \equiv \Theta\left[\frac{\Sigma \Lambda \Gamma}{1+q^{B} \phi_{q}}-\Phi(1-\beta \gamma)\right], \quad A_{21} \equiv-\frac{\Theta \Phi q^{B} \phi_{y}}{1+q^{B} \phi_{q}}, \quad$ and $A_{22} \equiv \frac{\Lambda \Gamma-A_{12} q^{B} \phi_{y}}{1+q^{B} \phi_{q}}$, with $\Theta \equiv \frac{1+q^{B} \phi_{q}}{1+\phi_{y}+q^{B} \phi_{q}}$ and $\Sigma \equiv \frac{1-\beta \gamma}{\beta \gamma}-\Phi\left(\frac{1-\beta \gamma}{1-\beta \gamma \Phi}\right) \phi_{q}$.

\footnotetext{
${ }^{50}$ Intuitively, a given change in the interest rate has a one-for-one effect on the anticipated percent change in the size of the bubble. How large that change is relative to output (i.e., how much it affects $\hat{q}_{t}^{B}$ ) depends on the initial size of the bubble relative to output.

${ }^{51}$ In the context of the two-period OLG model, Miao and Wang (2018) show that the finding in Galí (2014) regarding the impact of $\phi_{q}$ on the bubble volatility can be overturned if fluctuations in newly created bubbles are predictable and the equilibrium is unique. In that case, the aggregate bubble evolves according to
}

$$
\hat{q}_{t}^{B}=-\sum_{k=1}^{\infty}\left(\frac{\Lambda \Gamma}{1+q^{B} \phi_{q}}\right)^{k} E_{t}\left\{\hat{u}_{t+k}\right\},
$$

whose variance is decreasing in $\phi_{q}$ for any given arbitrary expectations $E_{t}\left\{\hat{u}_{t+k}\right\}$. 


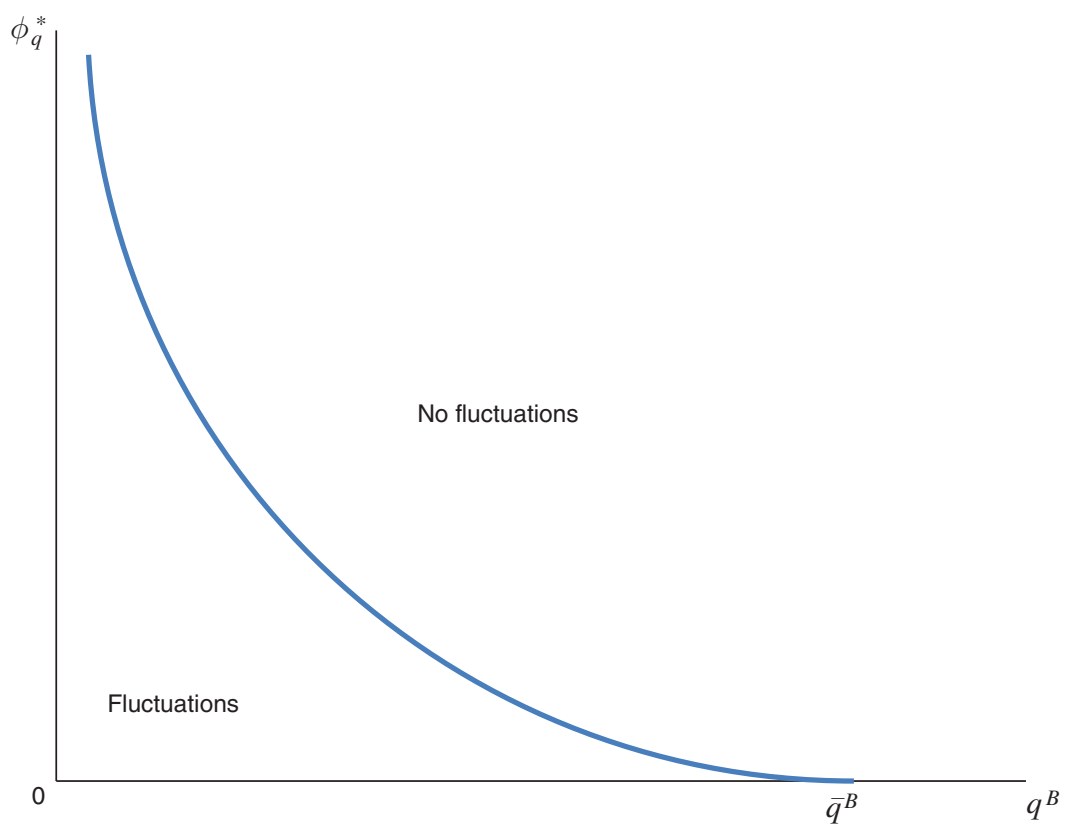

Figure 2. Bubble Fluctuations and LAB Policies

Note that as in all the cases considered above, the perfect foresight BGP is always $a$ solution to (52), corresponding to $\left[\hat{y}_{t}, \hat{q}_{t}^{B}\right]=[0,0]$ for all $t$. That solution is the only stationary if and only if both eigenvalues of matrix $\mathbf{A}$ lie within the unit circle (see, e.g., Blanchard and Kahn 1980). Otherwise, other solutions to (52) exist that involve stationary fluctuations in the aggregate bubble and the output gap as shown in the Appendix.

The necessary and sufficient conditions for both eigenvalues of a $2 \times 2$ matrix $\mathbf{A}$ to lie within the unit circle are given by $(\mathrm{a})|\operatorname{det}(\mathbf{A})|<1$ and (b) $|\operatorname{tr}(\mathbf{A})|$ $<1+\operatorname{det}(\mathbf{A}) .{ }^{52}$ Whether these conditions are satisfied or not in the case at hand depends in a nontrivial way on the interaction between different parameters, but some progress can be made in understanding the key factors by looking at some special cases.

Consider first the role of $\phi_{q}$, the bubble coefficient in the policy rule. As shown in the Appendix, for any $q^{B}>0$ and finite $\phi_{y} \geq 0, \lim _{\phi_{q} \rightarrow+\infty} \operatorname{det}(\mathbf{A})=0$ and $\lim _{\phi_{q} \rightarrow+\infty} \operatorname{tr}(\mathbf{A})=\Phi<1$. Given the continuity of $\operatorname{det}(\mathbf{A})$ and $\operatorname{tr}(\mathbf{A})$ with respect to $\phi_{q}$, it must be the case that the conditions for a unique solution are satisfied given a sufficiently large (but finite) $\phi_{q}$, independently of $\phi_{y}$. This result generalizes to an arbitrary $\phi_{y}$ coefficient the finding obtained above for $\phi_{y}=0$ and provides a theoretical justification for a sufficiently aggressive LAB policy as a way to rule out bubble-driven fluctuations.

Next, I study the role of $\phi_{y}$, the output coefficient in the policy rule. The desire to focus on a conventional stabilization motive may be motivated by the imperfect 
observability of the bubble and, hence, the risk that a LAB policy may generate unwarranted (and, hence, destabilizing) movements in interest rates. Can bubble-driven fluctuations be ruled out by a suitable choice of $\phi_{y}$, without the need of a systematic response to the bubble itself? Under $\phi_{q}=0$, the conditions for a unique solution to (52) corresponding to (a) and (b) above can be written, respectively, as

$$
\phi_{y}>\Phi \Lambda \Gamma-1 \equiv f\left(q^{B}\right)
$$

and

$$
\left(q^{B}-q_{*}^{B}\right)\left(\phi_{y}-h\left(q^{B}\right)\right)>0,
$$

where $h\left(q^{B}\right) \equiv \frac{(\Lambda \Gamma-1)(1-\Phi)}{1-\Psi}>0$ and, as above, $\Psi \equiv \Phi\left[1+(\Lambda \Gamma-1) \frac{1-\beta \gamma}{1-\beta \gamma \Phi}\right]$ is decreasing in $q^{B}$, with $q_{*}^{B}$ defined as the (unique) $q^{B}$ value for which $\Psi=1$. See Appendix for details.

As shown in the Appendix, $h\left(q^{B}\right)<f\left(q^{B}\right)$ for all $q^{B} \in\left[0, q_{*}^{B}\right]$, so there is no $\phi_{y}$ value which satisfies (53) and (54) simultaneously for that range of $q^{B}$ values. Thus, if the size of the bubble-output ratio along the BGP is smaller than $q_{*}^{B}$, bubble-driven fluctuations cannot be ruled out by means of an output gap-focused stabilization policy. On the other hand, $h\left(q^{B}\right)>f\left(q^{B}\right)$ for all $q^{B} \in\left(q_{*}^{B}, \bar{q}^{B}\right]$. Thus, over that range, the choice of $\phi_{y}>h\left(q^{B}\right)$ guarantees that (53) and (54) are both met, thus implying a unique solution to (52) and the absence of bubble-driven fluctuations. As shown in the Appendix, $h\left(q^{B}\right)$ is decreasing in $q^{B}$, with $\lim _{q_{*}^{B} \rightarrow q_{*}^{B}+} h\left(q^{B}\right)$ $=+\infty$ and $h\left(\bar{q}^{B}\right)=0$. Thus, the strength of the required output response in order to rule out bubble-driven fluctuations is decreasing in $q^{B}$, for $q^{B}>q_{*}^{B}$, becoming unbounded above as $q_{*}^{B} \rightarrow q_{*}^{B}+$. See Figure 3 for a graphical illustration of the previous findings.

The previous findings are related to the one obtained in the analysis of the flexible price economy, given that the equilibrium converges to its flexible price counterpart as $\phi_{y} \rightarrow+\infty$ and $\phi_{q} \rightarrow 0$. Thus, under that configuration of policy coefficients, bubble-driven fluctuations may emerge if and only if $q^{B} \in\left(q_{*}^{B}, \bar{q}^{B}\right]$ as in the flexible price equilibrium.

A Numerical Example.-While the previous model is clearly too stylized to be taken seriously quantitatively, it may be useful to get a sense of the ease with which bubble-driven fluctuations may emerge or be ruled out. With that purpose in mind, next I examine a simple numerical version of the OLG-NK model.

I start by assuming plausible settings for the different exogenous parameters $(\beta, \gamma, \Gamma$, and $v)$, taking the time unit to be a quarter. I set $\Gamma=1.004$, consistent with an average (annual) per capita GDP growth of 1.6 percent observed over the 1960-2016 sample period. To calibrate $\gamma$, I use the expected lifetime at age 16, which is 63.2 years in the United States, and thus set $\gamma=1-(1 /(4 \cdot 63.2)) \simeq 0.996$. I use the average employment ratio (relative to population aged 16 and over), which is (roughly) 0.6 on average over the period 1960-2016 as a proxy for $\alpha$. Conditional on the previous settings for $\alpha$ and $\gamma$, one can derive $v \simeq 0.997$. As discussed 


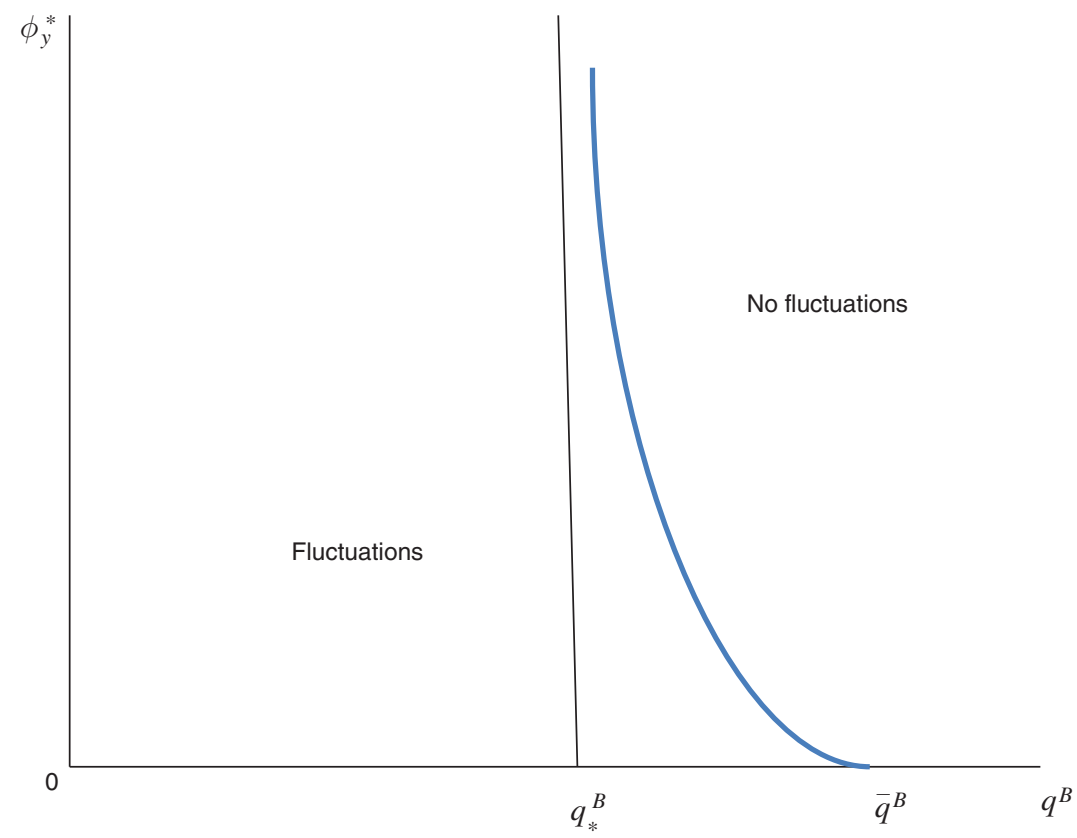

Figure 3. Bubble Fluctuations and Output Stabilization Policies

above, $\beta>v$ is required for the existence of a bubbly equilibrium. In contrast with the representative agent model, the OLG-NK model does not point to any empirical counterpart for $\beta$, though unity seems a "natural" upper bound for that parameter. For the sake of illustration, I set $\beta=0.998$, which is consistent with a (annual) real interest rate along a bubbleless $\mathrm{BGP}$ of 1.2 percent (i.e., $r_{0}=0.003$ ).

Under the previous calibration, a continuum of bubbly BGPs exists, with the upper bound on the bubble-output ratio given by $\bar{q}^{B} \simeq 23.6$, i.e., a bubble of size roughly 6 times annual output and with an associated range of (annual) interest rates between 1.2 and 1.6 percent. ${ }^{53}$ The threshold value $q_{*}^{B}$ which delimitates the regions with and without equilibria with bubble-driven fluctuations under flexible prices is given by $q_{*}^{B} \simeq 12.2$, roughly 3 times annual output. According to the analysis above, if the actual bubble-output ratio along the BGP were smaller than the previous threshold, a monetary policy that focused exclusively on output gap stabilization (i.e., without a LAB component) would not be able to rule out bubble fluctuations, though the central bank can always reduce the impact of the bubble on aggregate demand and output as much as needed by increasing $\phi_{y}$. In the limit, as $\phi_{y} \rightarrow+\infty$, the output gap would be fully stabilized and any fluctuations in the aggregate bubble would be described by the $\mathrm{AR}(1)$ process (41), with an autoregressive coefficient $\Psi^{-1}<1$ bounded below by a value close to 0.999 , the value of $\Psi^{-1}$ corresponding to $q^{B}=0$. Thus, any eventual aggregate bubble would display very high persistence in that case.

\footnotetext{
${ }^{53}$ Note that the average (annual) real interest rate on 3-month US Treasury bills over the period 1960-2016 has been roughly 1.4 percent (i.e., 0.35 percent quarterly), which lies within that interval.
} 
On the other hand, the monetary authority could eliminate bubble fluctuations and the resulting output gap fluctuations altogether by setting $\phi_{q}>\phi_{q}^{*}$, i.e., by pursuing a sufficiently aggressive LAB policy, even if $\phi_{y}=0$. As discussed above, the size of the threshold $\phi_{q}^{*}$ depends critically on $q^{B}$, the size of the bubble-output along the underlying BGP. Thus, in the numerical example considered here, if $q^{B}=4$ we have $\phi_{q}^{*}=0.0002$ so that the commitment to even a tiny response to the bubble would be enough to rule out fluctuations. The corresponding thresholds for $q^{B}=0.4, q^{B}=0.04$, and $q^{B}=0.004$ are, respectively, $\phi_{q}^{*}=0.00021, \phi_{q}^{*}=0.024$, and $\phi_{q}^{*}=0.24$, illustrating the strong nonlinearity in $\phi_{q}^{*}$ as a function of $q^{B}$ but also making it clear that a relatively weak LAB policy would be enough to rule out bubble-driven fluctuations (unless $q^{B} \rightarrow 0$ ).

\section{Bubbles and Welfare: Some Considerations}

The analysis of the OLG-NK model found in the previous sections, including the discussion of alternative policies, has focused on the conditions under which bubbles may exist along a BGP or the possibility of bubble-driven fluctuations. In this section, I make some considerations regarding the impact of any eventual bubbles on welfare. Following the structure of the analysis above, I start with a discussion of the connection between bubbles and welfare along a deterministic BGP, before turning to the potential impact of bubble-driven fluctuations on welfare.

\section{A. Bubbles and Welfare: BGP Analysis}

In the present section, I determine the expected lifetime utility at birth of an individual in the OLG-NK economy when the latter is moving along a deterministic BGP and study how that utility varies across BGPs when a multiplicity of the latter exists, indexed by the size of the bubble-output ratio $q^{B}$ (or, equivalently, by the interest rate). For the remainder of the paper, I maintain the assumption that $v<\beta$, which is necessary for the existence of bubbles in equilibrium.

Let $\mathcal{C}_{j}$ denote the consumption along a BGP of an individual of age $j$, normalized by productivity. Thus, we can write the period $t$ consumption of an individual from cohort $s \leq t$ as $C_{t \mid s}=\mathcal{C}_{t-s} \Gamma^{t}$. The expected lifetime utility at birth of an individual from cohort $s$ is thus given by

$$
\mathcal{U} \equiv \sum_{t=s}^{\infty}(\beta \gamma)^{t-s} \log C_{t \mid s}=\sum_{j=0}^{\infty}(\beta \gamma)^{j} \log \mathcal{C}_{j}+\Pi_{s},
$$

where $\Pi_{s} \equiv\left(\frac{\beta \gamma}{(1-\beta \gamma)^{2}}+\frac{s}{1-\beta \gamma}\right) \log \Gamma$ captures the impact of technical progress on lifetime utility across cohorts but is independent of the size of the bubble.

Given a discount factor $\Lambda \equiv 1 /(1+r)$ (which depends on $q^{B}$ ), the individual consumption Euler equation evaluated at a BGP implies

$$
\mathcal{C}_{j}=\left(\frac{\beta}{\Lambda \Gamma}\right)^{j} \mathcal{C}_{0}
$$


for $j=1,2,3, \ldots$ Using results derived in the Appendix, one can write an expression for $\mathcal{C}_{0}$ as a function of lifetime wealth:

$$
\mathcal{C}_{0}=(1-\beta \gamma)\left[\frac{u}{1-\gamma}+\left(\frac{1}{\alpha}\right)\left(\frac{1}{1-\Lambda \Gamma v \gamma}\right)\right] \mathcal{Y}=\frac{1}{1-\gamma}\left(1-\frac{\beta \gamma}{\Lambda \Gamma}\right) \alpha \mathcal{M}^{-\frac{1}{\varphi}}
$$

where the second equality can be derived, after some algebra, using (27) and (28) to substitute for $u$ as well as the definition of $\mathcal{Y}$. Combining (55), (56), and (57), one can write lifetime utility up to a multiplicative scalar and an exogenous additive term as

$$
\mathcal{U} \sim \log \left(1-\frac{\beta \gamma}{\Lambda \Gamma}\right)-\frac{\beta \gamma}{1-\beta \gamma} \log \Lambda
$$

Differentiating $\mathcal{U}$ with respect to $\Lambda$ and rearranging terms yields

$$
\frac{d \mathcal{U}}{d \Lambda}=-\frac{\beta \gamma}{(1-\beta \gamma) \Lambda}\left(\frac{\Lambda \Gamma-1}{\Lambda \Gamma-\beta \gamma}\right) \leq 0,
$$

where the inequality holds for all $\Lambda \Gamma \in[1, \beta / v]$, i.e., across all BGPs, while $d \mathcal{U} / d \Lambda=0$ if and only if $\Lambda \Gamma=1$, which corresponds to the BGP with $q^{B}=\bar{q}^{B}, u=0$, and $r=g$. Given the inverse relation between $q^{B}$ and $\Lambda$, it follows that lifetime utility across BGPs is increasing in the bubble-output ratio and attains a maximum when the latter takes its largest admissible value, $\bar{q}^{B}$. In addition, it follows that the bubbleless BGP is associated with the lowest lifetime utility. Note that for BGPs with $q^{B}<\bar{q}^{B}$, the interest rate is less than the growth rate of the economy; at the margin, this allows for an increase in lifetime utility through a reallocation of resources from the young to the old, which is what a bubble attains. Thus, and relative to other BGPs, the utility-maximizing BGP is associated with a relatively higher (lower) consumption for older (younger) cohorts.

\section{B. Bubble-Driven Fluctuations and Welfare}

Along a BGP, aggregate output and consumption evolve independently of the size of the bubble ratio, with the latter affecting lifetime utility only through the distribution of consumption across cohorts. By focusing on BGPs, the analysis in the previous subsection has abstracted from the possibility of fluctuations in the aggregate bubble and the resulting fluctuations in output and consumption discussed in Section IV.

What are the welfare implications of such bubble-driven fluctuations? For the sake of argument, consider first an environment in which lump-sum transfers are available and used by the fiscal authority to equate consumption across individuals (including the newly born), thus allowing us to abstract from distributional issues. Under our assumptions, the level of aggregate output and consumption along any BGP, given by $Y_{t}^{B G P}=\Gamma^{t} \alpha \mathcal{M}^{-\frac{1}{\varphi}}$, lies below the efficient level, $Y_{t}^{*}=\Gamma^{t} \alpha$, due to the monopolistic distortion in the goods market. Thus, and taking $Y_{t}^{B G P}$ as a starting point, any increase (decrease) in aggregate output and consumption resulting from a growing (shrinking) bubble has a first-order positive (negative) effect on 
utility. Furthermore, recurrent one-sided fluctuations in the bubble-and hence on output and consumption —of the sort generated by the Blanchard-type stochastic bubble analyzed in Section IV would also dominate from a welfare point of view the underlying BGP despite the likely volatility. On the other hand, recurrent symmetric bubble-driven fluctuations around a given BGP would lead to an unambiguous (second-order) welfare loss due to the curvature of the utility function, with the average utility loss per period given by $-(1 / 2) \operatorname{var}\left\{\hat{y}_{t}\right\}$.

In the absence of redistribution through lump-sum taxes, any welfare analysis should also take into account the distributional impact of bubble fluctuations. Such distributional effects are present even though complete financial markets have been assumed. The reason is that such markets are open only to cohorts that are currently alive, which effectively prevents the sharing of risks realized at any point in time (in particular, unexpected variations in existing or newly created bubbles) between newly born, incumbent, and future cohorts. Next, I discuss the key elements underlying the impact of bubble fluctuations on the three types of consumers.

Let $\hat{c}_{t \mid s} \equiv \log \left(C_{t \mid s} /\left(\mathcal{C}_{t-s} \Gamma^{t}\right)\right)$ denote the $\log$ deviation of $C_{t \mid s}$ from its value along the BGP. Consider first the impact of a positive bubble shock in period $t$ (i.e., $\varepsilon_{t}>0$ ) on the consumption path (relative to the BGP) of a newly born consumer $(s=t)$ :

$$
\frac{d \hat{c}_{t+k \mid t}}{d \varepsilon_{t}}=\frac{d \hat{c}_{t \mid t}}{d \varepsilon_{t}}+\sum_{j=0}^{k-1} \frac{d \hat{r}_{t+j}}{d \varepsilon_{t}}=\frac{(1-\beta \gamma) \Lambda \Gamma}{\Lambda \Gamma-\beta \gamma}\left[\frac{d \hat{u}_{t}}{d \varepsilon_{t}}+(1-v \gamma) \frac{d \hat{x}_{t}}{d \varepsilon_{t}}\right]+\sum_{j=0}^{k-1} \frac{d \hat{r}_{t+j}}{d \varepsilon_{t}},
$$

where the first equality follows from the consumer's Euler equation and the second equality makes use of the following result derived in the Appendix:

$$
\hat{c}_{t \mid t}=\frac{(1-\beta \gamma) \Lambda \Gamma}{\Lambda \Gamma-\beta \gamma}\left[\hat{u}_{t}+(1-v \gamma) \hat{x}_{t}\right]
$$

The effect of the bubble shock on the consumption path of an incumbent consumer $(s<t)$ is given by

$$
\frac{d \hat{c}_{t+k \mid s}}{d \varepsilon_{t}}=\frac{d \hat{c}_{t \mid *}}{d \varepsilon_{t}}+\sum_{j=0}^{k-1} \frac{d \hat{r}_{t+j}}{d \varepsilon_{t}}=\frac{(1-\beta \gamma) \Lambda \Gamma}{\beta \gamma}\left[\frac{d \hat{b}_{t}}{d \varepsilon_{t}}+v \gamma \frac{d \hat{x}_{t}}{d \varepsilon_{t}}\right]+\sum_{j=0}^{k-1} \frac{d \hat{r}_{t+j}}{d \varepsilon_{t}}
$$

for all $s<t$, where $\hat{c}_{t \mid *} \equiv \log \left(C_{t \mid *} /\left(\mathcal{C}_{*} \Gamma^{t}\right)\right)$, with $C_{t \mid *} \equiv(1 / \gamma)\left[C_{t}-(1-\gamma) C_{t \mid t}\right]$ denoting the average consumption among period $t$ "incumbent" consumers. The first equality exploits the fact that under the maintained assumption of complete markets, the consumption of all incumbent individuals will change in the same proportion in response to the shock (see (4)). Thus, $d \hat{c}_{t \mid s} / d \varepsilon_{t}=d \hat{c}_{t \mid *} / d \varepsilon_{t}$ for all $s<t$. The second equality makes use of the following expression derived in the Appendix:

$$
\hat{c}_{t \mid *}=\frac{(1-\beta \gamma) \Lambda \Gamma}{\beta \gamma}\left[\hat{b}_{t}+v \gamma \hat{x}_{t}\right] .
$$


Finally, the impact of the bubble shock on the consumption path of individuals born in the future $(s>t)$ is given by

$$
\frac{d \hat{c}_{s+k \mid s}}{d \varepsilon_{t}}=\frac{d \hat{c}_{s \mid s}}{d \varepsilon_{t}}+\sum_{j=0}^{k-1} \frac{d \hat{r}_{s+j}}{d \varepsilon_{t}}=\frac{(1-\beta \gamma) \Lambda \Gamma(1-v \gamma)}{\Lambda \Gamma-\beta \gamma} \frac{d \hat{x}_{s}}{d \varepsilon_{t}}+\sum_{j=0}^{k-1} \frac{d \hat{r}_{s+j}}{d \varepsilon_{t}}
$$

for all $s>t$, where I have used (59) to derive the second equality.

Thus, under the weak assumption that $d \hat{r}_{t+k} / d \varepsilon_{t} \geq 0$ for $k=0,1,2, \ldots$, a sufficient condition for a positive bubble shock to raise the consumption path (and hence welfare) of individuals currently alive, both newly born and incumbent $(s \leq t)$, is given by $d \hat{x}_{t} / d \varepsilon_{t}>0$; i.e., the positive bubble shock should cause an immediate increase in "fundamental" wealth. If, in addition, the latter effect is persistent, i.e., $d \hat{x}_{t+k} / d \varepsilon_{t}>0$ for $k=0,1,2,3, \ldots$, then a positive bubble shock will also raise the welfare of future cohorts. Note, on the other hand, that the exact distribution of the consumption (and welfare) gains resulting from a bubble expansion between the newly born and incumbent cohorts will depend on the extent to which the bubble shock takes the form of an innovation in the preexisting bubble $\left(d \hat{b}_{t} / d \varepsilon_{t}>0\right)$-in which case incumbent cohorts will draw a relatively larger benefit—or in newly created bubbles $\left(d \hat{u}_{t} / d \varepsilon_{t}>0\right)$-which benefit relatively more the newly born cohort. ${ }^{54}$

Under what conditions will fundamental wealth increase in response to a rising bubble? As shown in the Appendix, fundamental wealth $\hat{x}_{t}$ is related to the aggregate bubble through the difference equation

$$
\hat{x}_{t}=\chi E_{t}\left\{\hat{x}_{t+1}\right\}+\chi\left(\frac{1-\beta \gamma}{\beta \gamma \Phi}-\frac{(1-\beta \gamma) \phi_{y}+\phi_{q}}{1-\beta \gamma \Phi}\right) \hat{q}_{t}^{B},
$$

where $\chi \equiv \frac{\Phi}{1+\Phi\left(\frac{1-\beta \gamma}{1-\beta \gamma \Phi}\right) \phi_{y}} \in[0,1]$. Thus, any bubble shock that leads to a persistent increase in the aggregate bubble will raise current and future fundamental wealth (i.e., $d \hat{x}_{t+k} / d \varepsilon_{t}>0$ ) as long as the following condition is satisfied:

$$
(1-\beta \gamma) \phi_{y}+\phi_{q}<\frac{(1-\beta \gamma)(1-\beta \gamma \Phi)}{\beta \gamma \Phi},
$$

i.e., to the extent that the induced monetary policy response is not too strong.

The previous discussion has focused on the consequences of a positive bubble shock. The linearity of the equilibrium dynamics implies identical effects of negative bubble shocks but with the sign reversed. Accordingly, recurrent symmetric bubble-driven fluctuations around a BGP would only have a negative impact on (ex ante) expected lifetime utility due to the concavity of the utility function. That property would provide the main rationale for the kind of policies that rule out bubble fluctuations as analyzed in the previous section. Ex post, however, cohorts that are endowed with a sufficiently large bubble at birth may end up better off than

\footnotetext{
${ }^{54}$ The gains for future cohorts are independent of the nature of the bubble shock.
} 
in the underlying deterministic BGP despite the likely fluctuations in consumption over their lifetime.

\section{Concluding Comments}

The NK model remains the workhorse framework in macroeconomics, even though it is unsuitable - in its standard formulation-to accommodate the existence of asset price bubbles and, as a result, to address questions such as the conditions under which bubble-driven fluctuations may emerge or the interaction between aggregate bubbles and monetary policy. That shortcoming, however, is not tied to any essential ingredient of the model (e.g., nominal rigidities) but to the convenient (albeit unrealistic) assumption of an infinitely lived representative consumer. In the present paper, I have developed an extension of the basic NK model featuring overlapping generations, finite lives, and (stochastic) transitions to inactivity. That extension (which I referred to as OLG-NK, for short) allows, under certain conditions, for the existence of rational expectations equilibria with asset price bubbles. In particular, plausible calibrations of the model's parameters are consistent with the existence of a continuum of bubbly balanced growth paths as well as a bubbleless one (which always exists). When combined with sticky prices, fluctuations in the size of the aggregate bubble unrelated to changes in fundamentals have been shown to be a potential source of fluctuations in aggregate demand and output.

The analysis of the properties of the OLG-NK model yields several insights.

First, when one abstracts from the possibility of bubbly equilibria, the introduction of an overlapping generations structure does not change any of the qualitative properties of the standard NK model. In fact, the resulting equilibrium conditions describing the dynamics of inflation and the output gap are identical to those of a standard model with a "modified" discount factor that accounts for effectively shorter horizons. In particular, the presence of finite horizons by itself does not help overcome the so-called "forward guidance puzzle" uncovered by a number of authors in the context of an infinitely lived representative agent model.

Second, a "leaning against the bubble" (LAB) interest rate policy, if precisely calibrated, may succeed in insulating output and inflation from aggregate bubble fluctuations. Furthermore, if aggressive enough, the LAB policy may be able to rule out bubble fluctuations themselves. The strength of the required systematic policy response to the bubble, however, increases in an unbounded way as the average size of the bubble-output ratio approaches zero. On the other hand, a LAB policy which does not succeed in eliminating bubble fluctuations may end up increasing the volatility and persistence of bubble fluctuations as in Galí (2014).

Third, a policy that targets the output gap (or inflation) while ignoring the bubble will generally succeed at stabilizing those macro variables. If the average size of the bubble-output ratio lies above a certain threshold, a sufficiently aggressive output-based stabilization policy may rule out fluctuations in the bubble as well. On the other hand, if the average size of the bubble-output ratio lies below the same threshold, such a policy will not be able to eliminate bubble fluctuations, and their consequent randomization in distribution 
of resources across generations, even if they succeed in stabilizing output and inflation.

Finally, and in relation to the impact of bubbles on welfare, I have shown that expected lifetime utility along a deterministic balanced growth path is increasing in the associated bubble-output ratio. Secondly, an increase (decrease) in the size of the aggregate bubble raises (lowers) the expected lifetime utility of all cohorts as long as monetary policy doesn't "overreact" to it. However, and to the extent that consumers are risk averse, recurrent symmetric bubble-driven fluctuations will be welfare reducing and will thus justify policies that eliminate them.

Four additional remarks pointing to possible future research avenues are in order. Firstly, the analysis of the equilibrium dynamics above has assumed "rationality" of asset price bubbles. That assumption underlies the equilibrium conditions that individual and aggregate bubbles must satisfy, i.e., (20) and (21), respectively, and the implied log-linear approximation (33). However, the remaining equilibrium conditions, including the modified dynamic IS equations, are still valid even if the process followed by the aggregate bubble were to deviate from that of a rational bubble. That observation opens the door to analyses of the macroeconomic effects and policy implications of alternative specifications of the aggregate asset price misalignments.

Secondly, the analysis above suggests that in the absence of other frictions, bubble fluctuations by themselves do not generate a trade-off between output gap and inflation stabilization. In other words, the "divine coincidence" property holds. As a result, a literal interpretation of the model is likely to favor strict inflation targeting policies. It should be clear, however, that the model could be easily extended to incorporate some of the sources of policy trade-offs (e.g., staggered wage setting, shocks to desired markups, etc.) already found in the literature without altering or affecting the basic links between aggregate bubbles and economic activity.

Thirdly, the analysis in the present paper has been conducted using a framework that, like the textbook NK model, abstracts from endogenous capital accumulation. In the classical analysis of Tirole (1985) and others, however, where capital accumulation features prominently, a bubble expansion crowds out investment and through that channel leads to a subsequent decline in output. Note, however, that this would not necessarily be the case if capital accumulation were to be introduced in the OLG-NK model above since, in contrast with a Tirole-type model of bubbles, employment is endogenous and responsive to changes in aggregate demand (so that output can rise in response to a bubble expansion). As a result, an expansion in consumption does not mechanically imply a fall in investment. The latter's response will depend on the expected returns to investment (which may increase if aggregate demand increases persistently) and on the response of monetary policy (which is likely to reduce investment). Furthermore, and to the extent that firms' borrowing to finance investment is subject to collateral constraints, changes in the size of the aggregate bubble may affect the value of collateral and, through that channel, investment, potentially offsetting other crowding out channels. In that environment, a bubble may also distort the relative allocation of resources between consumption and investment, in addition to their levels, generating a trade-off for monetary policy 
that cannot be captured in a model without investment and financial frictions. All these considerations seem to call for an analysis of the potential role of investment in the transmission of bubble fluctuations to economic activity in an economy with nominal rigidities.

Fourthly, balanced growth paths characterized by a larger bubble-output ratio are associated with a higher real interest rate. Thus, the presence of a bubble along a BGP should make it less likely for the zero lower bound on the nominal interest rate to become binding, ceteris paribus and conditional on the bubble not bursting. Similarly, the bursting of the bubble would bring along a reduction in the natural rate of interest that could pull the interest rate toward the zero lower bound. The analysis of the interaction of bubble dynamics with the zero lower bound seems an additional avenue worth exploring in future research. 55

Note, finally, that the analysis of the equilibrium dynamics above has assumed that the central bank takes as given the BGP on which the economy settles and, hence, its associated real interest rate $r$ and bubble-output ratio $q^{B}$, both of which are parameters of the policy rule. But while the assumption of exogeneity of $r$ is a natural one in a context in which that variable is uniquely pinned down (e.g., the standard NK model), it is not obviously so in an economy like the one described by the OLG-NK model, in which a multiplicity of real interest rates and bubble-output ratios may, under certain conditions, be consistent with a perfect foresight BGP. In future research, I plan to explore the implications of relaxing the assumption of a policy invariant BGP, i.e., of allowing the central bank to play a role in determining the BGP where the economy settles.

\section{APPENDIX}

\section{A. Derivation of Consumption Functions}

The intertemporal budget constraint as of period $t$ for an individual born in period $s$ and still active in period $t \geq s$ can be derived by iterating (3) forward from $t$ onward to yield

$$
\sum_{k=0}^{\infty} \gamma^{k} E_{t}\left\{\Lambda_{t, t+k} C_{t+k \mid s}\right\}=A_{t \mid s}^{a}+\frac{1}{\alpha} \sum_{k=0}^{\infty}(\gamma v)^{k} E_{t}\left\{\Lambda_{t, t+k} W_{t+k} N_{t+k}\right\}
$$

For retired individuals born in period $s$, the corresponding constraint is

$$
\sum_{k=0}^{\infty} \gamma^{k} E_{t}\left\{\Lambda_{t, t+k} C_{t+k \mid s}\right\}=A_{t \mid s}^{r}
$$


Combining (4) with (60) and (61), we obtain the corresponding consumption functions

$$
\begin{aligned}
C_{t \mid s}^{a} & =(1-\beta \gamma)\left[A_{t \mid s}^{a}+\frac{1}{\alpha} \sum_{k=0}^{\infty}(\gamma v)^{k} E_{t}\left\{\Lambda_{t, t+k} W_{t+k} N_{t+k}\right\}\right] \\
C_{t \mid s}^{r} & =(1-\beta \gamma) A_{t \mid s^{*}}^{r}
\end{aligned}
$$

In particular, for a newly born consumer,

$$
\begin{aligned}
C_{t \mid t} & =(1-\beta \gamma)\left[\frac{U_{t}}{1-\gamma}+\frac{1}{\alpha} \sum_{k=0}^{\infty}(v \gamma)^{k} E_{t}\left\{\Lambda_{t, t+k}\left(W_{t+k} N_{t+k}+D_{t+k}\right)\right\}\right] \\
& =(1-\beta \gamma)\left[\frac{U_{t}}{1-\gamma}+\frac{1}{\alpha} \sum_{k=0}^{\infty}(v \gamma)^{k} E_{t}\left\{\Lambda_{t, t+k} Y_{t+k}\right\}\right] \\
& =(1-\beta \gamma)\left[\frac{U_{t}}{1-\gamma}+\frac{1}{\alpha} X_{t}\right] .
\end{aligned}
$$

Aggregating across all individuals and cohorts and imposing asset market clearing, we obtain the aggregate consumption function

$$
\begin{aligned}
C_{t} & =(1-\beta \gamma)\left[Q_{t}^{F}+Q_{t}^{B}+\sum_{k=0}^{\infty}(v \gamma)^{k} E_{t}\left\{\Lambda_{t, t+k} W_{t+k} N_{t+k}\right\}\right] \\
& =(1-\beta \gamma)\left[Q_{t}^{B}+\sum_{k=0}^{\infty}(v \gamma)^{k} E_{t}\left\{\Lambda_{t, t+k} Y_{t+k}\right\}\right] \\
& =(1-\beta \gamma)\left[Q_{t}^{B}+X_{t}\right] .
\end{aligned}
$$

Also note that one can evaluate (62) at a BGP to obtain the following expression for the consumption of a newly born individual, normalized by productivity, which is used in the section on welfare in the main text:

$$
\begin{aligned}
(63) \mathcal{C}_{0} & =(1-\beta \gamma)\left[\frac{u}{1-\gamma}+\left(\frac{1}{\alpha}\right)\left(\frac{1}{1-\Lambda \Gamma v \gamma}\right)\right] \mathcal{Y} \\
& =\frac{1-\beta \gamma}{1-\gamma}\left[\left(1-\frac{1}{\Lambda \Gamma}\right) \frac{\gamma(\beta-\Lambda \Gamma v)}{(1-\beta \gamma)(1-\Lambda \Gamma v \gamma)}+\frac{1-v \gamma}{1-\Lambda \Gamma v \gamma}\right] \mathcal{Y} \\
& =\frac{1}{(1-\gamma)(1-\Lambda \Gamma v \gamma)}\left[\left(1-\frac{1}{\Lambda \Gamma}\right)(\beta \gamma-\Lambda \Gamma v \gamma)+(1-\beta \gamma)(1-v \gamma)\right] \mathcal{Y} \\
& =\frac{1}{1-\gamma}\left(1-\frac{\beta \gamma}{\Lambda \Gamma}\right) \mathcal{Y},
\end{aligned}
$$

where the second equality makes use of (27) and (28) to substitute for $u$. 
B. Transversality Condition in a Bubbly BGP

The consumption function for an active individual born in period $s$ is

$$
C_{t+T \mid s}^{a}=(1-\beta \gamma)\left[A_{t+T \mid s}^{a}+\frac{W_{t+T} N / \alpha}{1-\Lambda \Gamma v \gamma}\right] .
$$

In particular,

$$
C_{t \mid s}^{a}=(1-\beta \gamma)\left[A_{t \mid s}^{a}+\frac{W_{t} N / \alpha}{1-\Lambda \Gamma v \gamma}\right]
$$

In addition, $C_{t+T \mid s}=(\beta / \Lambda)^{T} C_{t \mid s}$, thus implying

$$
A_{t+T \mid s}^{a}=(\beta / \Lambda)^{T}\left[A_{t \mid s}^{a}+\left(1-(\Lambda \Gamma / \beta)^{T}\right) \frac{W_{t} N / \alpha}{1-\Lambda \Gamma v \gamma}\right] .
$$

On the other hand, for a retired individual,

$$
C_{t \mid s}^{r}=(1-\beta \gamma) A_{t \mid s}^{r}
$$

Using the fact that $C_{t \mid s}^{r}=C_{t \mid s}^{a}$, we have

$$
A_{t \mid s}^{r}=A_{t \mid s}^{a}+\frac{W_{t} N / \alpha}{1-\Lambda \Gamma v \gamma} .
$$

The transversality condition for an active individual takes the form

$$
\begin{aligned}
\lim _{T \rightarrow \infty}(\gamma \Lambda)^{T} E_{t}\left\{A_{t+T \mid s}\right\}= & \lim _{T \rightarrow \infty}(\gamma \Lambda)^{T}\left[v^{T} A_{t+T \mid s}^{a}+\left(1-v^{T}\right) A_{t+T \mid s}^{r}\right] \\
= & \lim _{T \rightarrow \infty}(\gamma \Lambda)^{T}\left[A_{t+T \mid s}^{a}+\left(1-v^{T}\right) \Gamma^{T} \frac{W_{t} N / \alpha}{1-\Lambda \Gamma v \gamma}\right] \\
= & \lim _{T \rightarrow \infty}(\beta \gamma)^{T}\left[A_{t \mid s}^{a}+\left(1-(\Lambda \Gamma / \beta)^{T}\right) \frac{W_{t} N / \alpha}{1-\Lambda \Gamma v \gamma}\right] \\
& +\lim _{T \rightarrow \infty}(\gamma \Lambda \Gamma)^{T}\left(1-v^{T}\right) \frac{W_{t} N / \alpha}{1-\Lambda \Gamma v \gamma} \\
= & \frac{W_{t} N / \alpha}{1-\Lambda \Gamma v \gamma} \lim _{T \rightarrow \infty}\left[(\beta \gamma)^{T}-(\Lambda \Gamma \gamma)^{T}+(\Lambda \Gamma \gamma)^{T}-(\Lambda \Gamma v \gamma)^{T}\right] \\
= & 0,
\end{aligned}
$$

where the maintained assumption $\Lambda \Gamma v \gamma<1$ has been used. 


\section{Bubble-Driven Fluctuations: A Simple Representation}

Let the equilibrium be described by the system of difference equations

$$
\mathbf{x}_{t}=\mathbf{A} E_{t}\left\{\mathbf{x}_{t+1}\right\}
$$

where $\mathbf{x}_{t} \equiv\left[\hat{y}_{t}, \hat{q}_{t}^{B}\right]^{\prime}$. Let $\mathbf{A}$ have $q \leq 2$ eigenvalues with modulus less than one. If $q=2$, the stationary solution to (64) is unique and given by $\mathbf{x}_{t}=[0,0]$ for all $t$ (see, e.g., Blanchard and Kahn 1980). Here I focus on the case of $q<2$.

Consider the transformation $\mathbf{x}_{t}=\mathbf{Q} \mathbf{v}$, where $\mathbf{Q J} \mathbf{Q}^{-1}=\mathbf{A}$, where $\mathbf{J}$ is the canonical Jordan matrix and $\mathbf{Q} \equiv\left[\mathbf{q}^{(1)}, \mathbf{q}^{(2)}\right]$ is the matrix of generalized eigenvectors, corresponding to the two eigenvalues. Thus,

$$
\mathbf{v}_{t}=\mathbf{J} E_{t}\left\{\mathbf{v}_{t+1}\right\}
$$

Consider the case where $\mathbf{A}$ has eigenvalues $\left|\lambda_{1}\right|<1$ and $\left|\lambda_{2}\right|>1$. In that case, $\mathbf{J}=\left[\begin{array}{cc}\lambda_{1} & 0 \\ 0 & \lambda_{2}\end{array}\right]$. The stationary solutions to (65) take the form $\mathbf{v}_{1, t}=0$ and

$$
\mathbf{v}_{2, t}=\lambda_{2}^{-1} \mathbf{v}_{2, t-1}+\xi_{t}
$$

for all $t$, where $\xi_{t}$ is a (univariate) martingale difference process. Accordingly, $\quad \mathbf{x}_{t}=\mathbf{q}^{(2)} \mathbf{v}_{t}^{s}$ is the sunspot solution, or alternatively, $\mathbf{x}_{t}=\lambda_{2}^{-1} \mathbf{x}_{t-1}+\mathbf{q}^{(2)} \xi_{t}$. Conditional on $\left\{\xi_{t}\right\}$, that solution is unique up to normalization of $\mathbf{q}^{(2)}$.

Next, consider the case where $\mathbf{A}$ has eigenvalues $\left|\lambda_{1}\right|>1$ and $\left|\lambda_{2}\right|>1$. If both eigenvalues are real, $\mathbf{J}=\left[\begin{array}{cc}\lambda_{1} & 0 \\ 0 & \lambda_{2}\end{array}\right]$ and $\mathbf{q}^{(k)}$ corresponds to the eigenvector associated with eigenvalue $k$, for $k=1,2$. Otherwise, if $\lambda_{1}=a+b i$ and $\lambda_{2}=a-b i$ are complex conjugates, $\mathbf{J}=\left[\begin{array}{cc}a & -b \\ b & a\end{array}\right]$ and $\mathbf{q}^{(1)}$ and $\mathbf{q}^{(2)}$ are, respectively, the imaginary and real components of the eigenvector associated with the complex eigenvalues. In either case, (65) has a stable solution:

$$
\mathbf{v}_{t}=\mathbf{J}^{-1} \mathbf{v}_{t-1}+\xi_{t},
$$

where $\xi_{t}$ is a (bivariate) martingale difference process. Accordingly, and using $\mathbf{x}_{t}=\mathbf{Q} \mathbf{v}_{t}$ and $\mathbf{Q} \mathbf{J} \mathbf{Q}^{-1}=\mathbf{A}$, we have

$$
\mathbf{x}_{t}=\mathbf{A}^{-1} \mathbf{x}_{t-1}+\zeta_{t},
$$

where $\zeta_{t}=\mathbf{Q} \xi_{t}$. 
D. Conditions for Existence of Bubble-Driven Fluctuations: The General Case

It is easy to show, after some algebra, that

$$
\begin{aligned}
\operatorname{det}(\mathbf{A})= & \frac{\Phi \Lambda \Gamma}{1+\phi_{y}+q^{B} \phi_{q}}>0 \\
\operatorname{tr}(\mathbf{A})= & \Theta \Phi+\frac{\Lambda \Gamma}{1+q^{B} \phi_{q}}\left[1-\frac{\phi_{y}}{1+\phi_{y}+q^{B} \phi_{q}}\left(\frac{1-\Phi}{1-\beta \gamma \Phi}\right)\right] \\
& +\beta \gamma \Phi\left(\frac{\phi_{y}}{1+\phi_{y}+q^{B} \phi_{q}}\right)\left(\frac{1-\Phi}{1-\beta \gamma \Phi}\right)\left(1+\frac{\Lambda \Gamma}{1+q^{B} \phi_{q}} \frac{\phi_{q}}{1-\beta \gamma \Phi}\right)>0 .
\end{aligned}
$$

Note that for any $q^{B}>0, \lim _{\phi_{q} \rightarrow+\infty} \operatorname{det}(\mathbf{A})=0$ and $\lim _{\phi_{q} \rightarrow+\infty} \operatorname{tr}(\mathbf{A})$ $=\Phi<1$, thus establishing that a sufficiently aggressive LAB policy would rule out the possibility of bubble-driven fluctuations.

Next, consider the case of $\phi_{q}=0$. In that case, we can write

$$
\begin{aligned}
\operatorname{det}(\mathbf{A}) & =\frac{\Phi \Lambda \Gamma}{1+\phi_{y}}>0, \\
\operatorname{tr}(\mathbf{A}) & =\frac{\Phi}{1+\phi_{y}}+\Lambda \Gamma\left[1-\frac{\phi_{y}}{1+\phi_{y}}\left(\frac{1-\Phi}{1-\beta \gamma \Phi}\right)\right]+\beta \gamma \Phi\left(\frac{\phi_{y}}{1+\phi_{y}}\right)\left(\frac{1-\Phi}{1-\beta \gamma \Phi}\right) \\
& =\frac{\Phi}{1+\phi_{y}}+\Psi+\frac{1}{1+\phi_{y}}\left(\frac{1-\Phi}{1-\beta \gamma \Phi}\right)(\Lambda \Gamma-\beta \gamma \Phi)>0,
\end{aligned}
$$

where, as in the main text, $\Psi \equiv \Phi\left[1+(\Lambda \Gamma-1) \frac{1-\beta \gamma}{1-\beta \gamma \Phi}\right] \geq 0$, which is independent of $\phi_{y}$.

Accordingly, uniqueness condition $|\operatorname{det}(\mathbf{A})|<1$ can be written as

$$
\phi_{y}>\Phi \Lambda \Gamma-1 \equiv f\left(q^{B}\right)
$$

given $q^{B} \in\left[0, \bar{q}^{B}\right]$. On the other hand, condition $\operatorname{tr}(\mathbf{A})<1+\operatorname{det}(\mathbf{A})$ can be written as

$$
\begin{aligned}
\Psi-1< & \frac{1}{1+\phi_{y}}\left[\Phi(\Lambda \Gamma-1)-\left(\frac{1-\Phi}{1-\beta \gamma \Phi}\right)(\Lambda \Gamma-\beta \gamma \Phi)\right] \\
= & \frac{1}{1+\phi_{y}}\left[\Phi(\Lambda \Gamma-1)\left(\frac{1-\beta \gamma}{1-\beta \gamma \Phi}\right)+\Phi(\Lambda \Gamma-1) \frac{\beta \gamma(1-\Phi)}{1-\beta \gamma \Phi}\right. \\
& \left.-\left(\frac{1-\Phi}{1-\beta \gamma \Phi}\right)(\Lambda \Gamma-\beta \gamma \Phi)\right] \\
= & \frac{1}{1+\phi_{y}}[\Psi-1-(\Lambda \Gamma-1)(1-\Phi)] .
\end{aligned}
$$

Equivalently, and letting $h\left(q^{B}\right) \equiv(\Lambda \Gamma-1)(1-\Phi) /(1-\Psi)>0$, the previous inequality can be written more compactly as

$$
\left(q^{B}-q_{*}^{B}\right)\left(\phi_{y}-h\left(q^{B}\right)\right)>0
$$


given $q^{B} \in\left[0, \bar{q}^{B}\right]$.

Next, I analyze the conditions on $\phi_{y}$ that guarantee that (66) and (67) are jointly satisfied for any given $q^{B} \in\left[0, \bar{q}^{B}\right]$. As a preliminary step, it is useful to examine the properties of $f\left(q^{B}\right)$ and $h\left(q^{B}\right)$.

Note that $f\left(q^{B}\right)$ is continuous in $q^{B}$, with $f^{\prime}\left(q^{B}\right)<0, f(0)=(\beta / v)-1$ $>0$, and $f\left(\bar{q}^{B}\right)=(v / \beta)-1<0$. Furthermore, using the fact that 1 $=\Phi\left[1+(\Lambda \Gamma-1) \frac{1-\beta \gamma}{1-\beta \gamma \Phi}\right]$ at $q_{*}^{B}$, one can further show that $f\left(q_{*}^{B}\right)$ $=\frac{(\Lambda \Gamma-1) \beta \gamma(1-\Phi)}{1-\beta \gamma \Phi+(\Lambda \Gamma-1)(1-\beta \gamma)}>0$. For future reference, define $q_{0}^{B}$ such that $f\left(q_{0}^{B}\right)=0$, and note that $q_{*}^{B}<q_{0}^{B}<\bar{q}^{B}$.

On the other hand, $h\left(q^{B}\right)$ is continuous in $q^{B}$ except at $q^{B}$ $=q_{*}^{B}$, with $\left(q^{B}-q_{*}^{B}\right) h^{\prime}\left(q^{B}\right) \geq 0$ for $q^{B} \in\left[0, q_{*}^{B}\right) \cup\left(q_{*}^{B}, \bar{q}^{B}\right]$. Furthermore, $h(0)$ $=h\left(\bar{q}^{B}\right)=0, \lim _{q_{*}^{B} \rightarrow q_{*}^{B}-} h\left(q^{B}\right)=-\infty$ and $\lim _{q_{*}^{B} \rightarrow q_{*}^{B}+} h\left(q^{B}\right)=+\infty$.

The previous properties imply that $f\left(q^{B}\right)>h\left(q^{B}\right)^{*}$ for all $q^{B} \in\left[0, q_{*}^{B}\right)$ so that (66) and (67) cannot be satisfied simultaneously. Accordingly, equilibria with bubble-driven fluctuations will exist if $q^{B} \in\left[0, q_{*}^{B}\right]$, independently of the size of the output coefficient $\phi_{y}$.

On the other hand, we have $h\left(q^{B}\right)>f\left(q^{B}\right)$ for $q^{B} \in\left(q_{*}^{B}, \bar{q}^{B}\right]$. For $q^{B}>q_{0}^{B}$, this is clear since $f\left(q^{B}\right)<0$ and $h\left(q^{B}\right) \geq 0$ over that range. Furthermore, $h\left(q_{0}^{B}\right)$ $>f\left(q_{0}^{B}\right)=0$. Finally, it is easy to prove that $h\left(q^{B}\right)>f\left(q^{B}\right)$ for any $q^{B} \in$ $\left(q_{*}^{B}, q_{0}^{B}\right)$ as well. Suppose $h\left(q^{B}\right) \leq f\left(q^{B}\right)$. Then $(\Lambda \Gamma-1)(1-\Phi) \leq(\Phi \Lambda \Gamma-1)$ $\times(1-\Psi)$, where each term in brackets is strictly positive over the range $\left(q_{*}^{B}, q_{0}^{B}\right)$. But this is inconsistent with the fact that over the same range, $(1-\Psi)<(1-\Phi)$ and $(\Phi \Lambda \Gamma-1)<(\Lambda \Gamma-1)$. So, we must have $h\left(q^{B}\right)>f\left(q^{B}\right)$ over the range $\left(q_{*}^{B}, q_{0}^{B}\right)$ as well.

\section{E. Bubble-Driven Fluctuations and Welfare: Some Derivations}

As shown in Section A of the present Appendix, consumption of a newly born individual is given by

$$
C_{t \mid t}=(1-\beta \gamma)\left[\frac{1}{1-\gamma} U_{t}+\frac{1}{\alpha} X_{t}\right]
$$

Log-linearizing the previous expression around the BGP, and using the fact that $\mathcal{C}_{0} / \mathcal{Y}=\frac{1}{1-\gamma}\left(1-\frac{\beta \gamma}{\Lambda \Gamma}\right)$ as shown in $(63)$, yields

$$
\hat{c}_{t \mid t}=\frac{(1-\beta \gamma) \Lambda \Gamma}{\Lambda \Gamma-\beta \gamma}\left[\hat{u}_{t}+(1-v \gamma) \hat{x}_{t}\right],
$$

where $\hat{c}_{t \mid t} \equiv \log \left(C_{t \mid t} /\left(\mathcal{C}_{0} \Gamma^{t}\right)\right)$.

The average consumption of "incumbent" individuals, $C_{t \mid *}$, is given by

$$
C_{t \mid *} \equiv \frac{1}{\gamma}\left[C_{t}-(1-\gamma) C_{t \mid t}\right]=(1-\beta \gamma)\left[\frac{1}{\gamma} B_{t}+v X_{t}\right]
$$


Log-linearizing the previous expressions around the BGP yields

$$
\hat{c}_{t \mid *}=\frac{(1-\beta \gamma) \Lambda \Gamma}{\beta \gamma}\left[\hat{b}_{t}+v \gamma \hat{x}_{t}\right] \text {, }
$$

where $\hat{c}_{t \mid *} \equiv \log \left(C_{t \mid *} /\left(\mathcal{C}_{*} \Gamma^{t}\right)\right)$ and where use has been made of the fact that $\mathcal{C}_{*} / \mathcal{Y}=\beta /(\Lambda \Gamma)$.

Next, I analyze the dynamics of fundamental wealth, $\hat{x}_{t}$. From $(32)$ we have

$$
\hat{x}_{t}=\Phi E_{t}\left\{\hat{x}_{t+1}\right\}+\frac{1-\beta \gamma}{\beta \gamma} \hat{q}_{t}^{B}-\frac{\Phi}{1-\beta \gamma \Phi} \hat{r}_{t},
$$

which can be combined with (31) and (43) to obtain the following expression:

$$
\begin{aligned}
& \qquad \hat{x}_{t}=\chi E_{t}\left\{\hat{x}_{t+1}\right\}+\chi\left(\frac{1-\beta \gamma}{\beta \gamma \Phi}-\frac{(1-\beta \gamma) \phi_{y}+\phi_{q}}{1-\beta \gamma \Phi}\right) \hat{q}_{t}^{B}, \\
& \text { where } \chi \equiv \frac{\Phi}{1+\Phi\left(\frac{1-\beta \gamma}{1-\beta \gamma \Phi}\right) \phi_{y}} \in[0,1] .
\end{aligned}
$$

\section{REFERENCES}

Allen, Franklin, Gadi Barlevy, and Douglas M. Gale. 2017. "On Interest Rate Policy and Asset Bubbles." Federal Reserve Bank of Chicago Working Paper 2017-16.

Aoki, Kosuke, and Kalin Nikolov. 2015. "Bubbles, Banks and Financial Stability." Journal of Monetary Economics 74: 33-51.

Asriyan, Vladimir, Luca Fornaro, Alberto Martin, and Jaume Ventura. 2016. "Monetary Policy for a Bubbly World.” NBER Working Paper 22639.

Barlevy, Gadi. 2018. "Bridging between Policymakers' and Economists' Views on Bubbles." Economic Perspectives 42 (4): 1-21.

- Basco, Sergi. 2014. "Globalization and Financial Development: A Model of the Dot-Com and the Housing Bubbles." Journal of International Economics 92 (1): 78-94.

- Bengui, Julien, and Toan Phan. 2018. "Asset Pledgeability and Endogenously Leveraged Bubbles." Journal of Economic Theory 177: 280-314.

Bernanke, Ben S., and Mark Gertler. 1999. "Monetary Policy and Asset Price Volatility." In New Challenges for Monetary Policy, 77-128. Jackson Hole, WY: Federal Reserve Bank of Kansas City.

-Bernanke, Ben S., and Mark Gertler. 2001. "Should Central Banks Respond to Movements in Asset Prices?" American Economic Review 91 (2): 253-57.

Billi, Roberto, Jordi Galí, and Anton Nakov. 2020. "Bubbles and Liquidity Traps." Unpublished.

-Blanchard, Olivier Jean. 1979. "Speculative Bubbles, Crashes and Rational Expectations." Economic Letters 3 (4): 387-89.

- Blanchard, Olivier J. 1985. "Debt, Deficits, and Finite Horizons." Journal of Political Economy 93 (2): 223-47.

-Blanchard, Olivier. 2019. "Public Debt and Low Interest Rates." American Economic Review 109 (4): $1197-1229$.

Blanchard, Olivier Jean, and Charles M. Kahn. 1980. "The Solution of Linear Difference Models under Rational Expectations.” Econometrica 48 (5): 1305-11.

Bonchi, Jacopo. 2017. "Secular Stagnation and Rational Bubbles: How Bubbles Postponed the Great Recession." Unive rsity of Rome La Sapienza Working Paper 182.

Borio, Claudio, and Philip Lowe. 2002. "Asset Prices, Financial and Monetary Stability: Exploring the Nexus." BIS Working Paper 114. 
Caballero, Ricardo, and Alp Simsek. 2020. "A Risk-Centric Model of Demand Recessions and Speculation.” Quarterly Journal of Economics 135 (3): 1493-1566.

-Calvo, Guillermo A. 1983. "Staggered Prices in a Utility-Maximizing Framework." Journal of Monetary Economics 12 (3): 383-98.

- Carlstrom, Charles T., Timothy S. Fuerst, and Matthias Paustian. 2015. "Inflation and Output in New Keynesian Models with a Transient Interest Rate Peg." Journal of Monetary Economics 76: 230-43.

- Carvalho, Carlos, Andrea Ferrero, and Fernanda Nechio. 2016. "Demographics and Real Interest Rates: Inspecting the Mechanism.” European Economic Review 88: 208-26.

Del Negro, Marco, Marc Giannoni, and Christina Patterson. 2015. "The Forward Guidance Puzzle." Federal Reserve Bank of New York Staff Reports 574.

Dong, Feng, Jianjun Miao, and Pengfei Wang. 2018. "Asset Bubbles and Monetary Policy." http:// people.bu.edu/miaoj/working.htm.

-Farhi, Emmanuel, and Jean Tirole. 2011. "Bubbly Liquidity." Review of Economic Studies 79 (2): 678-706.

-Galí, Jordi. 2014. "Monetary Policy and Rational Asset Price Bubbles." American Economic Review 104 (3): 721-52.

Galí, Jordi. 2015. Monetary Policy, Inflation and the Business Cycle: An Introduction to the New Keynesian Framework and Its Application. 2nd ed. Princeton, NJ: Princeton University Press.

-Gertler, Mark. 1999. "Government Debt and Social Security in a Life-Cycle Economy." CarnegieRochester Conference Series on Public Policy 50: 61-110.

Hirano, Tomohiro, and Noriyuki Yanagawa. 2017. "Asset Bubbles, Endogenous Growth and Financial Frictions." Review of Economic Studies 84 (1): 406-43.

-Ikeda, Daisuke, and Toan Phan. 2019. "Asset Bubbles and Global Imbalances." American Economic Journal: Macroeconomics 11 (3): 209-51.

La Salle, J.P. 1976. The Stability of Dynamical Systems. Berlin, NJ: Hamilton Press.

-Martin, Alberto, and Jaume Ventura. 2012. "Economic Growth with Bubbles." American Economic Review 102 (6): 3033-58.

Martin, Alberto, and Jaume Ventura. 2018. "The Macroeconomics of Rational Bubbles: A User's Guide." Annual Review of Economics 10: 505-39.

McKay, Alisdair, Emi Nakamura, and Jón Steinsson. 2016. "The Power of Forward Guidance Revisited." American Economic Review 106 (10): 3133-58.

- Miao, Jianjun. 2014. "Introduction to Economic Theory of Bubbles." Journal of Mathematical Economics 53: 130-36.

- Miao, Jianjun, and Pengfei Wang. 2012. "Bubbles and Total Factor Productivity." American Economic Review 102: 82-87.

-Miao, Jianjun, and Pengfei Wang. 2014. "Sectoral Bubbles, Misallocation, and Endogenous Growth." Journal of Mathematical Economics 53: 153-63.

-Miao, Jianjun, and Pengfei Wang. 2018. "Asset Bubbles and Credit Constraints." American Economic Review 108 (9): 2590-2628.

- Miao, Jianjun, Zhouxiang Shen, and Pengfei Wang. 2019. "Monetary Policy and Rational Asset Price Bubbles: Comment." American Economic Review 109 (5): 1969-90.

- Nisticò, Salvatore. 2012. "Monetary Policy and Stock-Price Dynamics in a DSGE Framework." Journal of Macroeconomics 34 (1): 126-46.

-Piergallini, Alessandro. 2006. "Real Balance Effects and Monetary Policy." Economic Inquiry 44 (3): 497-511.

-Samuelson, Paul A. 1958. "An Exact Consumption-Loan Model of Interest with or without the Social Contrivance of Money." Journal of Political Economy 66 (6): 467-82.

-Santos, Manuel S., and Michael Woodford. 1997. "Rational Asset Pricing Bubbles." Econometrica 65 (1): 19-57.

- Taylor, John B. 2014. "The Role of Policy in the Great Recession and the Weak Recovery." American Economic Review 104 (5): 61-66.

- Tirole, Jean. 1985. “Asset Bubbles and Overlapping Generations.” Econometrica 53 (6): 1499-1528.

Woodford, Michael. 2003. Interest and Prices: Foundations of a Theory of Monetary Policy. Princeton: Princeton University Press.

-Woodford, Michael. 2011. "Simple Analytics of the Government Spending Multiplier." American Economic Journal: Macroeconomics 3 (1): 1-35.

-Yaari, Menahem E. 1965. "Uncertain Lifetime, Life Insurance, and the Theory of the Consumer." Review of Economic Studies 32 (2): 137-50. 